Max-Planck-Institut für demografische Forschung

Max Planck Institute for Demographic Research

Konrad-Zuse-Strasse 1 - D-18057 Rostock - GERMANY

Tel +49 (0) 3812081 - 0; Fax +49 (0) 3812081 - 202;

http://www.demogr.mpg.de

MPIDR WORKING PAPER WP 2012-004

JANUARY 2012 (UPDATED JANUARY 2013)

\title{
Perturbation analysis of indices of lifespan variability
}

Alyson A. van Raalte (vanraalte@demogr.mpg.de)

Hal Caswell

This working paper has been approved for release by: Mikko Myrskylä (myrskyla@demogr.mpg.de), Head of the Max Planck Research Group on Lifecourse Dynamics and Demographic Change.

(C) Copyright is held by the authors.

Working papers of the Max Planck Institute for Demographic Research receive only limited review. Views or opinions expressed in working papers are attributable to the authors and do not necessarily reflect those of the Institute. 


\title{
Perturbation analysis of indices of lifespan variability
}

\author{
Alyson A. van Raalte ${ }^{1,2}$, Hal Caswell ${ }^{3,1}$ \\ ${ }^{1}$ Max Planck Institute for Demographic Research, Rostock, Germany \\ ${ }^{2}$ Department of Public Health, Erasmus MC, Rotterdam, the Netherlands \\ ${ }^{3}$ Woods Hole Oceanographic Institution, Woods Hole MA, USA
}

\begin{abstract}
A number of indices exist to calculate lifespan variation, each with different underlying properties. Here we present new formulae for the response of seven of these indices to changes in the underlying mortality schedule (life disparity, the Gini coefficient, the standard deviation, the variance, Theil's index, the mean logarithmic deviation, and the inter-quartile range). We derive each of these indices from an absorbing Markov chain formulation of the life table, and use matrix calculus to obtain the sensitivity and the elasticity (i.e., the proportional sensitivity) to changes in age-specific mortality. Using empirical French and Russian male data we compare the underlying sensitivities to mortality change under different mortality regimes in order to test under which conditions the indices might differ in their conclusions about the magnitude of lifespan variation. Finally, we demonstrate how the sensitivities can be used to decompose temporal changes in the indices into contributions of age-specific mortality changes. The result is an easily computable method for calculating the properties of this important class of longevity indices.
\end{abstract}




\section{Introduction}

In this paper we present a detailed comparative study of the sensitivity and elasticity of indices of variation in longevity. The longevity experience of a cohort has long been summarized by its expectation, but recently attention has expanded to focus on variation in longevity as a natural complement to describing the average length of life. Indices of variability have been compared across populations to measure the rectangularity of the survival curve or degree of mortality compression for both human and and non-human populations (see for instance Eakin and Witten 1995; Edwards and Tuljapurkar 2005; Smits and Monden 2009; van Raalte et al. 2011; Vaupel, Zhang and van Raalte 2011). They have also been employed above the modal age at death to examine whether old-age mortality is being compressed, or whether these deaths are shifting to higher ages (Brown et al. 2012; Cheung et al. 2005; Cheung and Robine 2007; Kannisto 2000; 2001; Ouellette and Bourbeau 2011; Thatcher et al. 2010). The various indices of lifespan variation have been compared by Anand et al. (2001); Cheung et al. (2005); Kannisto (2000); Shkolnikov, Andreev and Begun (2003); Vaupel et al. (2011) and Wilmoth and Horiuchi (1999). These authors have found such high correlations among indices as to make them apparently interchangeable.

Our concern here is with a neglected aspect of these indices: their response to perturbations of the underlying mortality schedule. In general, perturbation analysis calculates the change in some quantity $x$ due to changes in one or more parameters $\theta$ from which that quantity is calculated. This can be expressed as a derivative, the sensitivity $d x / d \theta$, which gives the response of $x$ to an additive perturbation in $\theta$, or as the proportional derivative, or

elasticity $\frac{x}{\theta} \frac{d x}{d \theta}$, which gives the proportional change in $x$ resulting from a proportional change in $\theta$.

Sensitivity analysis has a long history in formal demography. Examples include the sensitivity of the intrinsic growth rate $r$ or $\lambda$ to changes in mortality and fertility, first derived by Hamilton (1966), followed by Demetrius (1969), Keyfitz (1971), and then in general form by Caswell (1978). The sensitivity of life expectancy to changes in mortality was derived by Keyfitz (1971), and investigated in more detail by Pollard $(1982 ; 1988)$ and more recently by Caswell (2006) and Wrycza and Baudisch (2012). Keyfitz introduced an elasticity of life expectancy in terms of life table entropy (see Keyfitz and Caswell 
2005; chap. 4), which was further developed by Goldman and Lord (1986); Vaupel (1986) and Vaupel and Canudas Romo (2003). The sensitivity of the stable population structure to changes in mortality and fertility was investigated numerically by Coale (1972) and analytically by Caswell (2001; 2008). The sensitivity of short-term projections was analyzed by Caswell (2007). All of these sensitivity analyses quantify the effect of parameter changes, permit rigorous comparison of different perturbations, and provide the tools for decomposing changes in an index into components arising from changes in each parameter (Caswell 1989; 2001). Until its perturbation analysis is available, our understanding of any index is incomplete. Indices of variation in longevity are no exception, but no general perturbation theory is available.

The patterns of sensitivity and elasticity of each index will reveal whether the correlations among the values of the various indices extend to their response to changes in mortality. This includes analytically determining the age ranges over which mortality reductions lead to increases or decreases in variation. These age ranges are separated by a "threshold age," at which the derivative is zero. Perturbation results can also be used to target age-specific mortality reductions to ages which best reduce lifespan variation. Moreover, because the sensitivity results are age-specific, they can be related to normative concepts of inequality or social preference for actions designed to reduce mortality at particular ages (Asada 2007; Anand et al. 2001; Gakidou, Murray and Frenk 2000). This has rarely been done; a notable exception to this is the WHO attempt to quantify inequality over individuals as part of World Health Report 2000, using an index similar to a Gini coefficient, modified by expert opinion (Gakidou et al. 2000; Gakidou and King 2002; WHO 2000).

In this paper, we provide, for the first time, readily computable formulae for the sensitivities and elasticities of seven of the most commonly used indices to changes in age-specific mortality and, by extension, to any parameter[s] affecting age-specific mortality. Our approach is to reformulate the problem of lifespan variability in terms of an absorbing Markov chain, and to use methods from matrix calculus (Caswell 2008; 2009; 2010). We will show how to use the results to decompose differences among populations or over time into contributions from changes in age-specific mortality, using Life Table Response Experiment (LTRE) methods (Caswell 2001). We apply these methods to data from France and Russia, and illustrate instances where these different sensitivities cause indices to disagree on the magnitude or even direction of changes in lifespan variation. 


\section{Indices of lifespan variation}

We compare the following indices of variability:

(1) Life disparity $\left(e^{\dagger}\right)$ : Life disparity is a life table based index, defined as the average remaining life expectancy at death, or alternatively the average years of life lost in a population due to death. The elasticity of life expectancy with respect to mortality change, also known as Keyfitz' $H$ (Keyfitz 1977), is $e^{\dagger}$ divided by the life expectancy at birth (Goldman and Lord 1986; Vaupel 1986; Vaupel and Canudas Romo 2003).

(2) Gini coefficient $(G)$ : The Gini coefficient is often used in economic inequality research. It ranges from 0 to 1 , with higher numbers signaling greater inequality. It is the mean of the absolute value of the inter-individual differences in age at death, divided by the life expectancy (Shkolnikov et al. 2003).

$(3,4)$ Theil's index $(T)$ and the mean logarithmic deviation $(M L D)$ : Both $T$ and $M L D$ are based on the entropy of the distribution of age at death, developed from information theory by Henry Theil in the 1960s (Theil 1967). The entropy of a distribution measures the amount of information needed to specify the result of sampling; if everyone died at the same age, no information would be needed and the entropy-based measures would be zero.

(5-7) Standard deviation $(S)$, variance $(V)$, and inter-quartile range $(I Q R)$ of the distribution of age at death: The indices $S, V$ and $I Q R$ are standard statistical measures of variability applied to the distribution of age at death.

Different research objectives often call for the use of one index over another due to their underlying formal properties. The variance $V$, Theil index $T$ and the mean logarithmic deviation $M L D$ are all additively decomposable into between- and within-group variation (Shorrocks 1980; van Raalte 2011). This decomposition can be used to study the contribution of between-group differences to the total level of lifespan variation. The Gini coefficient $G$ can also be decomposed in this way, but contains an overlap term (Lambert 
and Aronson 1993). The $M L D$ index can additionally be additively decomposed over time, to account for compositional change to the between- and within-group variation components (Mookherjee and Shorrocks 1982). The life disparity index $e^{\dagger}$ has interesting connections to the perturbation theory of life expectancy. The product of $e^{\dagger}$ and the average rate of progress in reducing age specific death rates is equal to the rate of change in life expectancy (Vaupel and Canudas Romo 2003).

Indices also differ in whether they measure absolute inequality (the level of variation would be unaffected by additive gains to everyone's lifespan) or relative inequality (the level of variation would be unaffected by proportional gains to everyone's lifespan). Additive indices are more easily interpretable, as they are normally expressed in years.

The sensitivity of indices to changes in mortality at different ages is an important and poorly understood property of the indices. In some circumstances, society might consider variability in ages at death caused by high levels of premature mortality to be more detrimental than variability caused by differences in old age mortality. In such a case, usage of an index with a high sensitivity to early death would emphasize the response to the mortality viewed as most important. As economist Paul Allison (1978) noted: "The choice of an inequality measure is properly regarded as a choice among alternative definitions of inequality rather than a choice among alternative ways of measuring a single theoretical construct."

The seven indices we examine here are highly correlated across countries and times. Some of these correlations have been reported by Vaupel et al. (2011) and Wilmoth and Horiuchi (1999). We present the correlations among all seven indices, from birth and from age 10 (Table 1), calculated over all female and male life tables ${ }^{1}$ currently in the Human Mortality Database HMD (2012). Thus we expect that all of them will pick up most of the general patterns in lifespan variation in inter-population comparisons. Our focus is on the details of the response of the indices to changes in mortality.

\footnotetext{
${ }^{1}$ We excluded populations that were double-counted, i.e. we excluded civilian population in favour of total national population life tables, and excluded nationally aggregated life tables in favour of including regional or ethnic-based life tables.
} 


\begin{tabular}{l|ccccccc}
\hline & $e^{\dagger}$ & $G$ & $T$ & $M L D$ & $S$ & $V$ & $I Q R$ \\
\hline$e^{\dagger}$ & 1.000 & & & & & & \\
$G$ & 0.978 & 1.000 & & & & & \\
$T$ & 0.947 & 0.991 & 1.000 & & & & \\
$M L D$ & 0.965 & 0.991 & 0.992 & 1.000 & & & \\
$S$ & 0.981 & 0.933 & 0.893 & 0.930 & 1.000 & & \\
$V$ & 0.987 & 0.945 & 0.911 & 0.944 & 0.996 & 1.000 & \\
$I Q R$ & 0.967 & 0.966 & 0.948 & 0.956 & 0.920 & 0.944 & 1.000 \\
\hline & $e_{10}^{\dagger}$ & $G_{10}$ & $T_{10}$ & $M L D_{10}$ & $S_{10}$ & $V_{10}$ & \multirow{2}{*}{$I Q R_{10}$} \\
\hline$e_{10}^{\dagger}$ & 1.000 & & & & & & \\
$G_{10}$ & 0.986 & 1.000 & & & & & \\
$T_{10}$ & 0.978 & 0.995 & 1.000 & & & & \\
$M L D_{10}$ & 0.979 & 0.990 & 0.995 & 1.000 & & & \\
$S_{10}$ & 0.986 & 0.962 & 0.961 & 0.973 & 1.000 & & \\
$V_{10}$ & 0.984 & 0.964 & 0.971 & 0.980 & 0.998 & 1.000 & \\
$I Q R_{10}$ & 0.981 & 0.978 & 0.977 & 0.976 & 0.958 & 0.966 & 1.000 \\
\hline
\end{tabular}

Table 1: Pearson correlation coefficients between pairs of indices, calculated from birth (ages $0-110+$ ) in the top panel and calculated conditional upon survival to age 10 (ages 10-110+) in the bottom panel, for all female and male life tables in the Human Mortality Database (7516 in total). 


\section{Markov chain formulations of longevity}

To develop perturbation results, we formulate the mortality schedule as a finite-state absorbing Markov chain (Caswell 2001; 2006; 2009; 2010; Feichtinger 1973). This formulation lets us express the various indices in matrix notation, and then apply matrix calculus to obtain the sensitivity and elasticity of each index to changes in parameters (e.g. Caswell 2006; 2009; 2011). Since this study focuses on human demography, we focus on the ageclassified model. Nevertheless, these results could be generalized to apply to stage-classified populations.

Notation. We use matrix notation in deriving the sensitivities. Matrices are denoted by upper case bold faced symbols (e.g., $\mathbf{X}$ ) and vectors by lower case bold faced symbols $(\mathbf{x})$; vectors are column vectors by default, and $\mathbf{x}^{\top}$ denotes the transpose of $\mathbf{x}$. The symbol diag $(\mathbf{x})$ denotes the matrix with the vector $\mathbf{x}$ on the diagonal and zeros elsewhere. The vector $\mathbf{e}$ is a vector of ones, and the vector $\mathbf{e}_{i}$ is the $i$ th unit vector; i.e., the vector with a 1 in the $i$ th location and zeros elsewhere. The Hadamard, or element-by-element product is denoted by $\circ$ and the Kronecker product by $\otimes$. The vec operator (e.g., vec $\mathbf{X}$ ) stacks the columns of a matrix into a column vector. See Caswell (2001) or Keyfitz and Caswell (2005) for an account of matrix models in demography.

We consider $s$ age classes. Let $\mathbf{U}$ be a matrix $(s \times s)$ with survival probabilities on the subdiagonal and zeros elsewhere; i.e.,

$$
u_{i+1, i}=1-q_{i-1} \quad i=1, \ldots, s-1
$$

where $q_{i}$ is the probability of death between ages $i$ and $i+1$ from the life table. Note that matrix entries are indexed starting at 1 , but age in the life table (conventionally) starts at 0 . Thus, for example, the probability of surviving from the first to the second age state is the complement of the probability of death between age 0 and age 1 , or $u_{2,1}=1-q_{0}$.

The matrix $\mathbf{U}$ describes transitions among the transient states in the Markov chain. Death is an absorbing state; we classify deaths by the age class at death with a diagonal matrix $\mathbf{M}(s \times s)$, where

$$
m_{i, i}=1-q_{i-1} \quad i=1, \ldots, s
$$


The transition matrix ${ }^{2}$ for the Markov chain is

$$
\mathbf{P}=\left(\begin{array}{c|c}
\mathbf{U} & 0 \\
\hline \mathbf{M} & \mathbf{I}
\end{array}\right)
$$

where 0 is a matrix of zeros and $\mathbf{I}$ is an $(s \times s)$ identity matrix.

In this Markov chain, absorption corresponds to death, and the time to absorption corresponds to longevity. The statistical properties of longevity can be directly calculated from $\mathbf{P}$. The mean time spent in age class $i$, conditional on starting in age class $j$ is given by the $(i, j)$ entry of the fundamental matrix

$$
\mathbf{N}=(\mathbf{I}-\mathbf{U})^{-1}
$$

Because absorption corresponds to death, the time to absorption can be treated as a measure of longevity (Caswell 2001, 2006, 2009). The mean time to absorption is given by the column sums of $\mathbf{N}$. Let $\tilde{\boldsymbol{\eta}}$ denote the vector whose $i$ th entry is the expected time to absorption for an individual in age class $i$; it is given by

$$
\tilde{\boldsymbol{\eta}}^{\top}=\mathbf{e}^{\top} \mathbf{N}
$$

where $\mathbf{e}$ is a vector of ones. However, it can be shown that this exceeds by 0.5 years the life expectancy calculated by the usual life table formulations; accordingly, we use

$$
\boldsymbol{\eta}=\tilde{\boldsymbol{\eta}}-0.5 \mathbf{e}
$$

to represent life expectancy. The subtraction of the constant 0.5 does not affect the calculations of sensitivities.

The vector of variances in longevity satisfies

$$
\mathbf{v}^{\top}=\mathbf{e}^{\top} \mathbf{N}(2 \mathbf{N}-\mathbf{I})-\boldsymbol{\eta}^{\top} \circ \boldsymbol{\eta}^{\top}
$$

where o denotes the Hadamard, or element-by-element product.

The complete distribution of age at death, conditional on starting in age class $j$, is given by column $j$ of the matrix

$$
\mathrm{B}=\mathrm{MN}
$$

\footnotetext{
${ }^{2}$ Note that $\mathbf{P}$ is column-stochastic and operates on column vectors, to agree with the orientation of population projection matrices (e.g., Caswell 2001, Keyfitz and Caswell 2005).
} 
The distribution of age at death for an individual in the first age class is given by the first column of $\mathbf{B}$;

$$
\mathbf{f}=\mathbf{B e}_{1}
$$

The survivorship function $\ell$, beginning at age 1 and with a radix $\ell(0)=1$, is given by

$$
\ell=\mathbf{e}-\mathbf{C f}
$$

where

$$
\mathbf{C}=\left(\begin{array}{cccc}
0 & 0 & \cdots & 0 \\
1 & 0 & \cdots & 0 \\
\vdots & \vdots & & \vdots \\
1 & 1 & 1 & 0
\end{array}\right)
$$

takes cumulative sums of the vector $\mathbf{f}$.

The vector $\mathbf{x}$ contains the average age at death in the age interval (i.e. for French males in 2005 it is $\{0.06,1.5,2.5, \ldots, 109.5,111.32\})$.

In Table 2 we present the conventional lifetable notation alongside the less familiar matrix notation for each index. In conventional notation $\ell_{y}$ is survivorship, $d_{y}$ the death density, and $e_{y}$ remaining life expectancy for the age interval $y$ to $y+1$. We further denote $a_{y}$ as the length of the age interval lived by those who died. An overbar, for example $\bar{e}_{y}$, is used when adjustments to the variable are necessary to account for the portion of the age interval lived by those who died, i.e.

$$
\bar{e}_{y}=e_{y}+a_{y}\left(e_{y+1}+e_{y}\right)
$$

By this same logic, $\bar{x}_{y}$ is the average age at death over the interval. Generally it is the age halfway in between the two age intervals, but in the first year of life $\bar{x}_{0}=a_{0}$. The highest age interval is denoted by $\omega$.

Finally in the $I Q R$ formula, $\hat{x}_{1}$ and $\hat{x}_{3}$ are the interpolated first and third age quartiles, at which 25 and 75 percent of the total deaths have occurred.

\section{Sensitivity and elasticity analysis}

Expressing longevity in terms of an absorbing Markov chain and applying matrix calculus has greatly expanded the possibilities for perturbation anal- 


\begin{tabular}{l|cc}
\hline & conventional LT notation & matrix notation \\
\hline$e^{\dagger}$ & $\sum_{y=0}^{\omega} d_{y} \bar{e}_{y}$ & $\mathbf{f}^{\top} \boldsymbol{\eta}$ \\
$T$ & $1-\frac{1}{e_{0}} \sum_{y=0}^{\omega} \ell_{y+1}^{2}$ & $1-\frac{1}{\eta_{1}} \mathbf{e}^{\boldsymbol{\top}}[(\mathbf{e}-\mathbf{C f}) \circ(\mathbf{e}-\mathbf{C f})]$ \\
$M L D$ & $\sum_{y=0}^{\omega} d_{y}\left(\frac{\bar{x}_{y}}{e_{0}} \ln \frac{\bar{x}_{y}}{e_{0}}\right)$ & $\mathbf{f}^{\boldsymbol{\top}}\left[\left(\frac{\mathbf{x}}{\eta_{1}}\right) \circ\left(\log \frac{\mathbf{x}}{\eta_{1}}\right)\right]$ \\
$V$ & $\sum_{y=0}^{\omega} d_{y}\left(\ln \frac{e_{0}}{\bar{x}_{y}}\right)$ & $\mathbf{f}^{\top}\left[\log \left(\eta_{1}\right) \mathbf{e}-\log \mathbf{x}\right]$ \\
$S Q R$ & $\sum_{y=0}^{\omega} d_{y}\left(\bar{x}_{y}-e_{0}\right)^{2}$ & {$\left[\mathbf{e}^{\top} \mathbf{N}(2 \mathbf{N}-\mathbf{I})-\boldsymbol{\eta}^{\top} \circ \boldsymbol{\eta}^{\top}\right]^{\top}$} \\
& & $\sqrt{V}$ \\
$\hat{x}_{3}-\hat{x}_{1}$ & $\hat{x}_{3}-\hat{x}_{1}$ \\
\hline
\end{tabular}

Table 2: Formulae for calculating indices in conventional life table formulation (discrete, assuming $l_{0}$ of 1 ) and their equivalent formulation in matrix notation. 
ysis (Caswell 2001; 2008; 2009; 2010; Willekens 1977). To assess the absolute and proportional effects on the indices of changes in the underlying mortality rates we needed the analytic expressions for the sensitivity and elasticity of the seven indices of lifespan variability with respect to mortality. The sensitivity of $e^{\dagger}$ was first derived by Zhang and Vaupel (2009) in an ageclassified model, which was further developed by Wagner (2010). This was later generalized to an age and stage classified model by Caswell (2010), who also derived expressions for the sensitivity and elasticity of the variance and the standard deviation (Caswell 2009). The other expressions were newly derived for this paper.

As described in detail in Section 9.1, the sensitivity of a $n \times 1$ vector $\mathbf{y}$ to a $m \times 1$ vector of parameters $\boldsymbol{\theta}$ is given by the $n \times m$ matrix

$$
\frac{d \mathbf{y}}{d \boldsymbol{\theta}^{\top}}=\left(\frac{d y_{i}}{d \theta_{j}}\right),
$$

whose $(i, j)$ entry is the derivative of $y_{i}$ with respect to $\theta_{j}$. The elasticity of $\mathbf{y}$ to $\boldsymbol{\theta}$ is

$$
\frac{\epsilon \mathbf{y}}{\epsilon \boldsymbol{\theta}^{\boldsymbol{\top}}}=\operatorname{diag}(\mathbf{y})^{-1} \frac{d \mathbf{y}}{d \boldsymbol{\theta}^{\boldsymbol{\top}}} \operatorname{diag}(\boldsymbol{\theta}) .
$$

The sensitivities and elasticities of each index to age-specific mortality were derived using matrix calculus (Magnus and Neudecker 1988). These techniques are given extensive treatment in recent papers by Caswell, using most of the same notation that we have here (Caswell 2008; 2009; 2010). The derivation of the sensitivities of all the indices to mortality can be found in the appendix. We performed all numerical calculations in MATLAB (version 7.3.0), and include both MATLAB and R code as an online appendix. The formulae resulting from the matrix calculus may appear complicated, but the complication arises from, and accounts for, the network of interactions among the variables, and they are easily computed.

We now turn to the demographic applications, especially in comparing the sensitivities of these indices, examining how they have changed over time as we have moved from high to low mortality regimes, and using the sensitivities as a decomposition method. 


\section{A comparison of sensitivities: France and Russia}

We used French male data to broadly illustrate the underlying sensitivities and elasticities of each index. We calculated the indices under four very different mortality regimes: high mortality (1888), medium mortality (1948), low mortality (2005) and war/epidemic year (1918). The last regime is interesting, in that the distribution of age at death has a second mode around young adulthood, and a long right tail instead of a long left tail. To help visualize these differences, all four distributions are plotted in Figure 1.

\section{French male death densities}

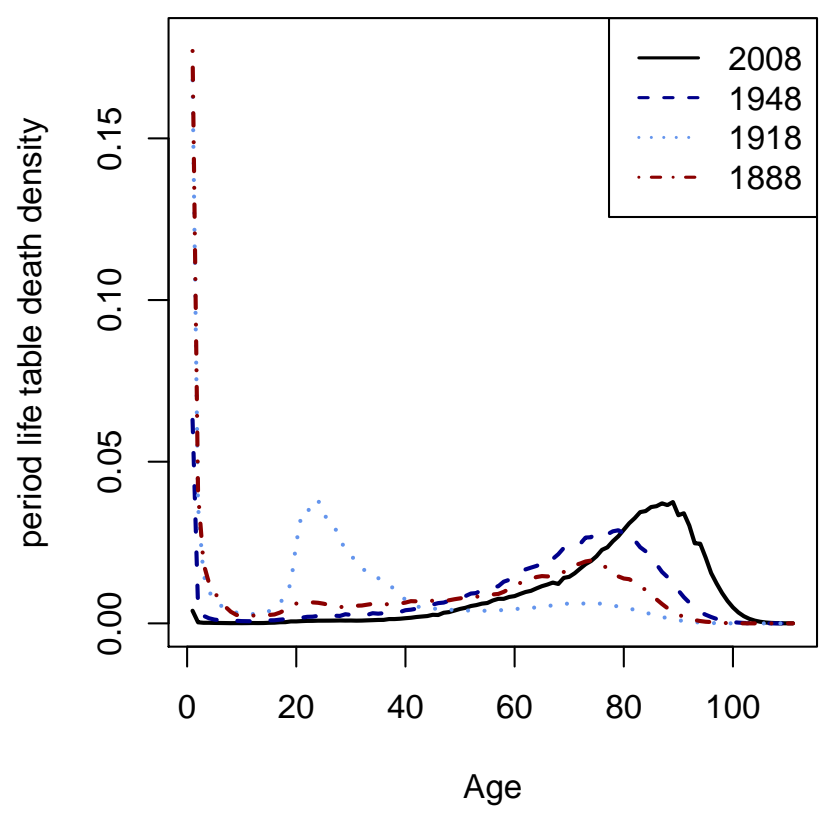

Figure 1: The French male death densities for which the sensitivities and elasticities of the indices are compared

All indices are highly sensitive to changes in infant mortality. For this reason we calculated the sensitivities and elasticities both at birth (Figures 2 and 3) and at age 10 (see appendix). Given the different units for each index, the elasticities are perhaps intuitively easier to interpret. The y axis measures the percent change in the index from a one percent change in mortality at 
each age on the $\mathrm{x}$ axis.

As we would expect from the high correlations between indices, the sensitivities follow similar general age patterns. The primary differences are in the sensitivity to infant mortality, the slope of the decline from birth to late adulthood, and in the age at which the sensitivities cross the $\mathrm{x}$-axis (this age is the same for $S$ and $V$ ). This age has been termed the threshold age or $a^{\dagger}$ due to its original derivation for the $e^{\dagger}$ index (Zhang and Vaupel 2009). Reductions in mortality below the threshold age reduce lifespan variation, while reductions above the threshold age increase the variation. Since it is the age where the derivative is zero, it can be calculated using either the sensitivity or elasticity formulae in the appendix. The threshold age has increased over time, and the differences between threshold ages of the indices have considerably diminished.

In general, conditioning upon survival to age 10 resulted in only minor changes to the pattern of the sensitivity of each index to age-specific mortality, although it did remove some of the differences between indices found when examined from birth, see appendix Figure 1. This was particular the case for the $M L D$ and $T$ indices, which are so highly sensitive to changes at birth that changes at other ages are largely masked. The $I Q R$ index produces the most unique sensitivity patterns. It is only sensitive to transfers between quartiles and not to transfers within quartiles. Transfers of course are an awkward concept in mortality research, particularly as there are no finite life years that need to be distributed within the population. But in practice the idea of age rationing in health care, sacrificing facilities and medicine for older individuals to save younger individuals, comes close.

We imagined a scenario of targeted interventions leading to mortality reduction, to examine how the different sensitivity profiles of the various indices could affect our assessment of whether a population is becoming more egalitarian in its ages at death. Using French male data from 1888 and 2008, we calculated the threshold age and the percentage change in the index from a 10 percent decrease in death rates over selected age ranges. This was done for the indices calculated at birth and conditional upon survival to age 10 (Table 3). The largest differences between the indices occurred for mortality change at the youngest age ranges, particularly for the 1888 age-at-death distribution where early death was more common. In the modern distribution, differences between indices were also large over the middle adult age 

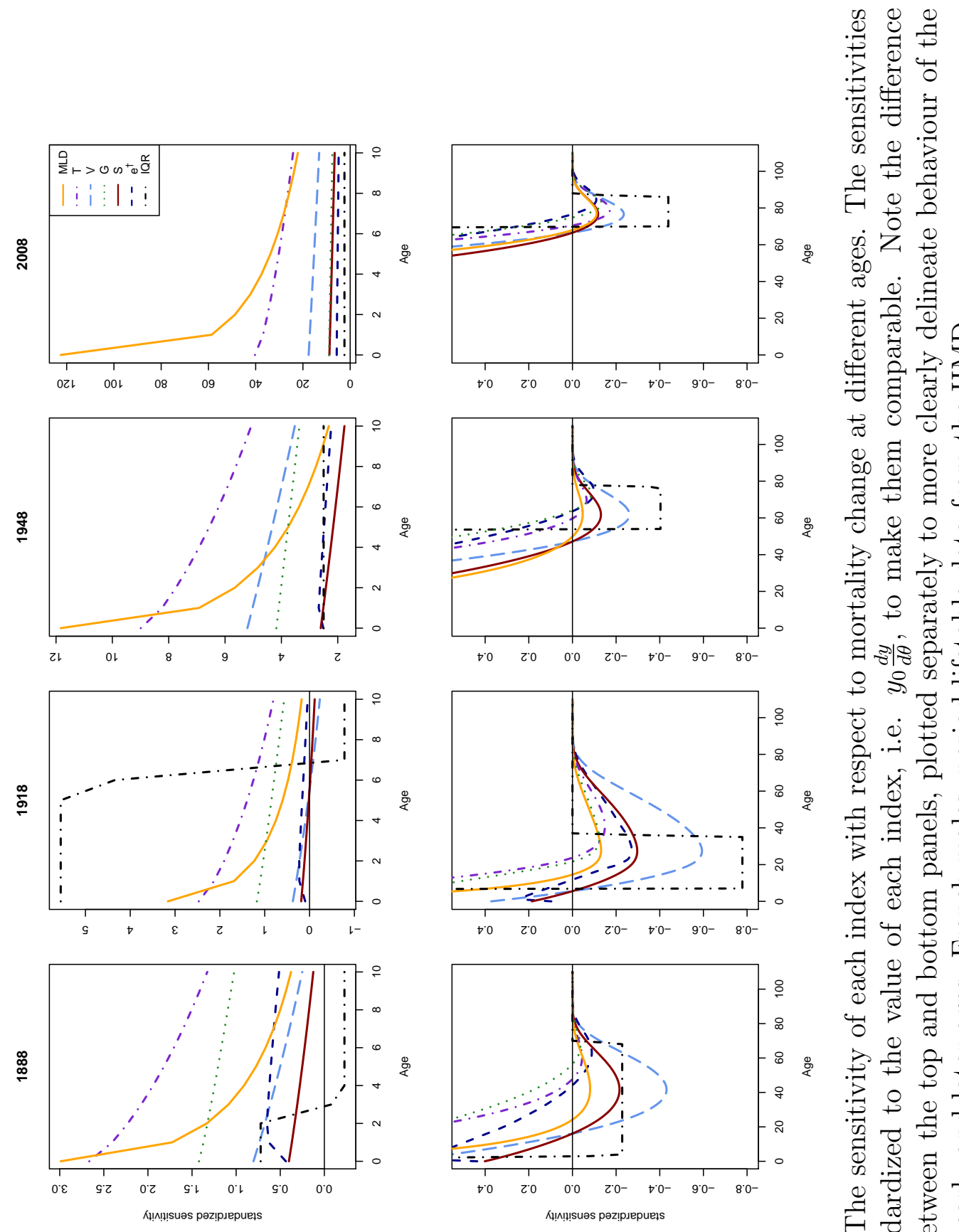

๘ $\stackrel{0}{0}$

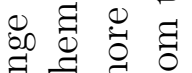

矛

ن

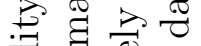

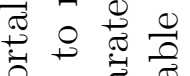

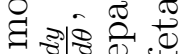

$\circ \stackrel{5}{\circ}$

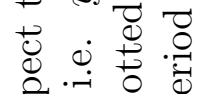

की

过

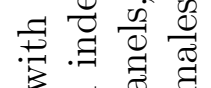

$\beta$ ฮี ป

造

붕

당 이얻

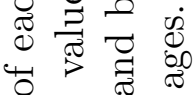

현

.

च Ð

क.

E恶莕

ㄱ

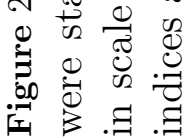



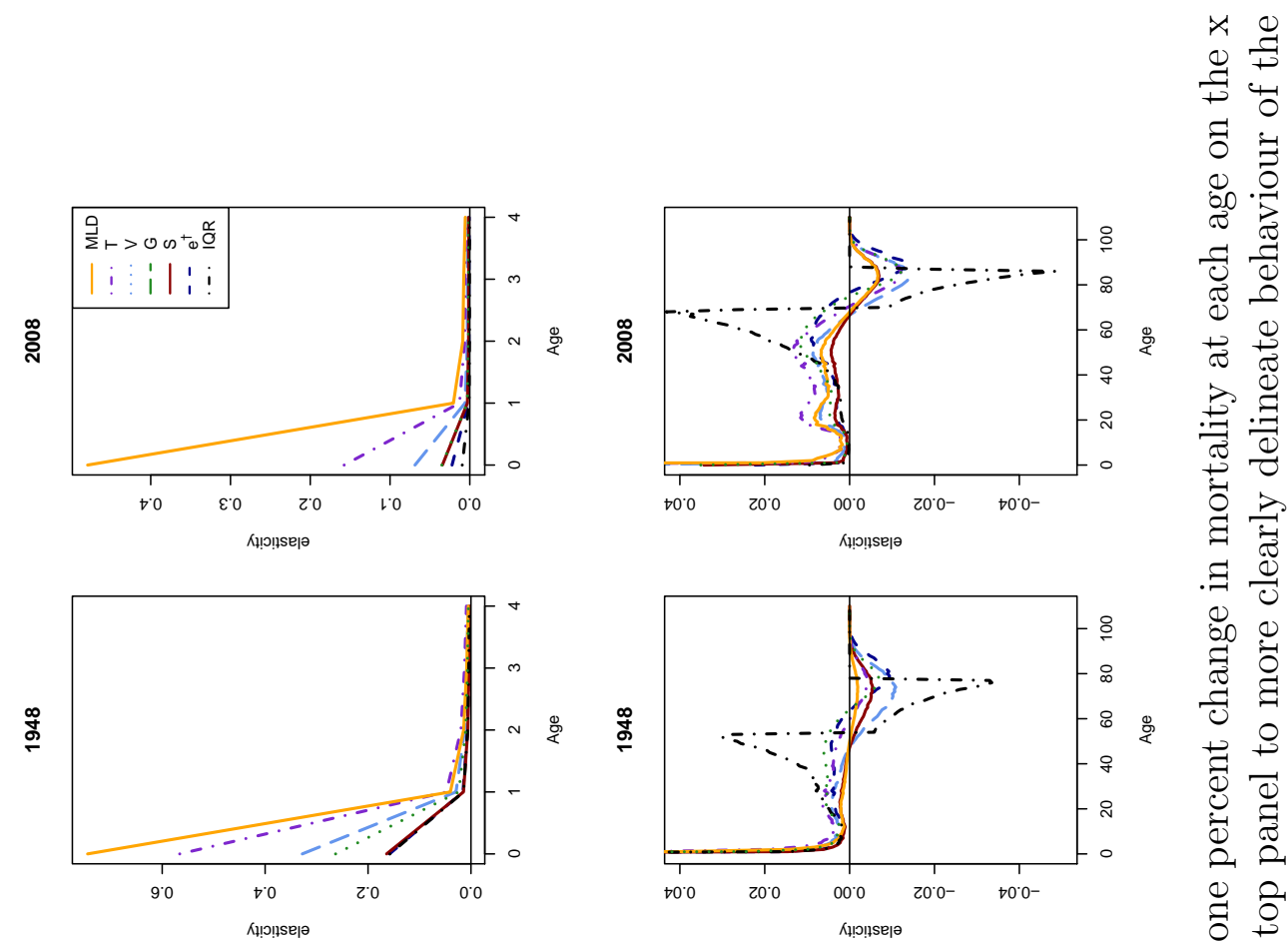

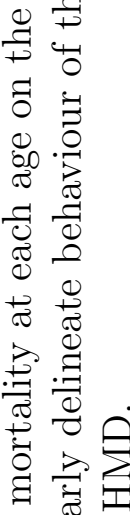
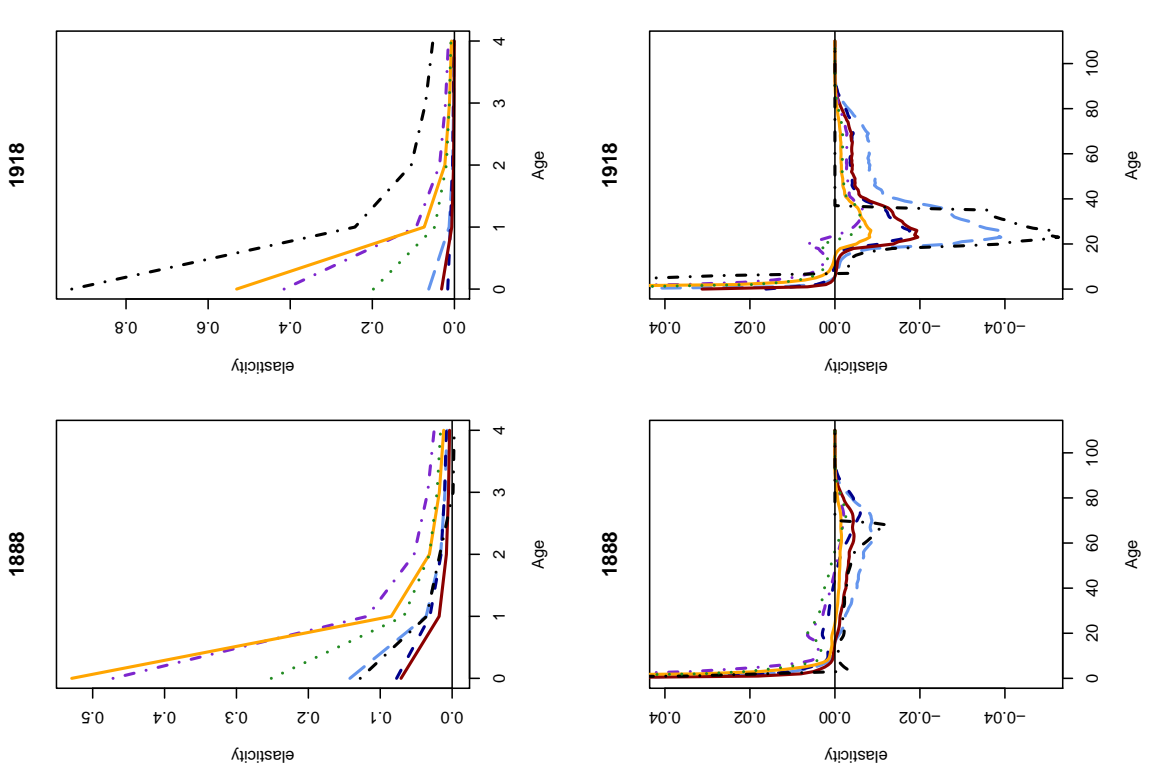

$\exists$

So

胥

荘 $\stackrel{\sigma}{*}$

○

颉

웡

$\Phi$

ఠ

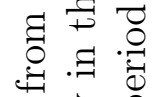

$x \geq 2$

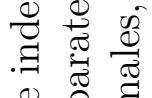

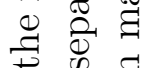

Ð ठ

80 茫

帘 0 के

त

궁

$+80$

유요

范

可

$\ddot{\theta} \approx$

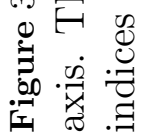


range 60-80. Indices with younger threshold ages such as $M L D, S$, and $V$ found that mortality reduction over these ages increased lifespan variation, while the other indices all measured a decrease in variability. The different responses to mortality change over adult ages between using an index calculated at birth or at age 10 was large for the historic population, but made relatively little difference for the modern population.

\begin{tabular}{|c|c|c|c|c|c|c|c|c|c|c|}
\hline \multirow[b]{3}{*}{ Index } & \multirow{2}{*}{\multicolumn{2}{|c|}{ Threshold Age }} & \multicolumn{8}{|c|}{ Percent increase in index from mortality reduction at ages } \\
\hline & & & \multicolumn{2}{|c|}{$0-5$} & \multicolumn{2}{|c|}{$20-40$} & \multicolumn{2}{|c|}{$60-80$} & \multicolumn{2}{|c|}{$85+$} \\
\hline & 1888 & 2008 & 1888 & 2008 & 1888 & 2008 & 1888 & 2008 & 1888 & 2008 \\
\hline$e^{\dagger}$ & 43.4 & 76.2 & -2.7 & -0.3 & -0.3 & -0.6 & 1.0 & -1.0 & 0.2 & 2.1 \\
\hline$G$ & 56.9 & 74.9 & -4.6 & -0.4 & -0.8 & -0.9 & 0.4 & -0.9 & 0.0 & 1.2 \\
\hline$I Q R$ & 2.8 & 69.7 & -3.8 & -0.1 & 0.4 & -0.5 & 1.1 & -2.7 & 0.0 & 1.6 \\
\hline$M L D$ & 24.8 & 68.7 & -6.2 & -5.1 & 0.1 & -1.2 & 0.3 & 0.2 & 0.0 & 0.9 \\
\hline$S$ & 16.6 & 67.1 & -1.4 & -0.6 & 0.3 & -0.8 & 0.9 & 0.3 & 0.0 & 0.9 \\
\hline$T$ & 46.3 & 69.7 & -8.0 & -1.9 & -0.6 & -1.9 & 0.4 & -0.2 & 0.0 & 1.2 \\
\hline$V$ & 16.6 & 67.1 & -2.8 & -1.1 & 0.6 & -1.6 & 1.9 & 0.6 & 0.1 & 1.9 \\
\hline \multirow[t]{3}{*}{ Range } & 54.1 & 9.1 & 6.6 & 5.0 & 1.4 & 1.4 & 1.6 & 3.4 & 0.2 & 1.2 \\
\hline & \multirow{2}{*}{\multicolumn{2}{|c|}{ Threshold Age }} & \multirow{2}{*}{\multicolumn{8}{|c|}{$\begin{array}{l}\text { Percent increase in index from mortality reduction at ages } \\
10-15\end{array}$}} \\
\hline & & & & & & & & & & \\
\hline Index & 1888 & 2008 & 1888 & 2008 & 1888 & 2008 & 1888 & 2008 & 1888 & 2008 \\
\hline$e_{10}^{\dagger}$ & 57.2 & 76.7 & -0.4 & 0.0 & -1.8 & -0.7 & 1.5 & -1.0 & 0.3 & 2.2 \\
\hline$G_{10}$ & 58.8 & 75.1 & -0.6 & -0.1 & -2.7 & -1.0 & 0.9 & -1.1 & 0.1 & 1.3 \\
\hline$I Q R_{10}$ & 43.1 & 69.8 & -0.5 & 0.0 & -4.2 & -0.4 & 2.8 & -2.8 & 0.0 & 1.7 \\
\hline$M L D_{10}$ & 45.5 & 68.6 & -1.6 & -0.3 & -3.3 & -2.7 & 1.7 & 0.2 & 0.1 & 2.0 \\
\hline$S_{10}$ & 42.5 & 67.2 & -0.4 & -0.1 & -1.2 & -1.0 & 1.5 & 0.3 & 0.1 & 1.0 \\
\hline$T_{10}$ & 50.5 & 70.5 & -1.3 & -0.2 & -3.8 & -2.4 & 1.7 & -0.2 & 0.1 & 1.5 \\
\hline$V_{10}$ & 42.5 & 67.2 & -0.9 & -0.2 & -2.5 & -1.9 & 3.1 & 0.5 & 0.2 & 2.1 \\
\hline Range & 16.3 & 9.5 & 1.3 & 0.3 & 3.0 & 2.3 & 2.2 & 3.4 & 0.3 & 1.1 \\
\hline
\end{tabular}

Table 3: The threshold age (columns 2 and 3) and the percentage increase in each index resulting from a 10 percent reduction in mortaly over the given age ranges, for indices calculated from birth (top panel) and conditional upon survival to age 10 (bottom panel). Results obtained by reducing mortality by $10 \%$ at each age, using French male period lifetable data from Human Mortality Database, and then re-computing the indices. 


\section{Decomposition of temporal trends in vari- ability}

Our perturbation results make it possible to decompose differences or changes in an index into contributions from differences or changes in any of the parameters. The approach is known in population biology as Life Table Response Experiment (LTRE) analysis, and has been widely used (see review in Caswell 2001, Chapter 10). It applies to any demographic statistic for which the sensitivity to the underlying vital rates can be calculated. The version used here is closely related to the decomposition method independently derived and described by Horiuchi, Wilmoth and Pletcher (2008).

Here, we use LTRE analysis to decompose temporal changes in the indices of lifespan variability into contributions from changes in age-specific mortality rates, for Russian males from 1958 to 2006.

Let $y$ be an index, $\boldsymbol{\theta}$ a vector of parameters (mortality rates in our application) and let $t$ denote time. The decomposition proceeds from noting that, to first order,

$$
y(t+\Delta t) \approx y(t)+\frac{d y}{d \boldsymbol{\theta}^{\top}} \frac{d \boldsymbol{\theta}}{d t} \Delta t
$$

The product of the two derivatives in (15) gives the overall change in $y$ due to the changes in all the parameters over the interval $\Delta t$. Thus the contributions to that change are given by the entries of the vector

$$
\mathbf{c}(t)=\left(\frac{d y}{d \boldsymbol{\theta}^{\top}}\right)^{\top} \circ\left(\frac{d \boldsymbol{\theta}}{d t}\right)
$$

These contributions can be integrated over time to obtain the contributions to the change in $y$ from $t_{0}$ to $t_{1}$,

$$
y\left(t_{1}\right) \approx y\left(t_{0}\right)+\sum_{i=t_{0}}^{t_{1}} \mathbf{c}(i)
$$

We computed the rate of change in the parameters, $d \boldsymbol{\theta} / d t$, using the MATLAB function gradient, which uses a central difference algorithm to compute the derivatives.

The sequence of age-specific mortality changes experienced by Russian males (Anand et al. 2001; Shkolnikov et al. 2003) provides an interesting example 
of decomposition analysis. From 1958 to 2006, infant mortality declined substantially, from around 47 to 12 deaths per thousand live births. This decline was particularly rapid from 1958-1968. At the same time, adult mortality, especially between ages 40 and 50 years, fluctuated a great deal. Adult mortality increased slowly but steadily until the mid-1980s, then declined rapidly between 1984 and 1987 following the anti-alcohol campaigns, and then increased steeply with the mortality crisis brought on by the upheavals of transition to a market economy (Leon et al. 1997).

Figure 4 shows each index relative to its level in 1959, calculated from birth. Apart from the $I Q R$, all indices show that lifespan variation decreased during the period, with large fluctuations in the interim. The standard deviation $S$ and the variance $V$ showed less volatility than the other indices while $T, G$ and $I Q R$ showed the most.

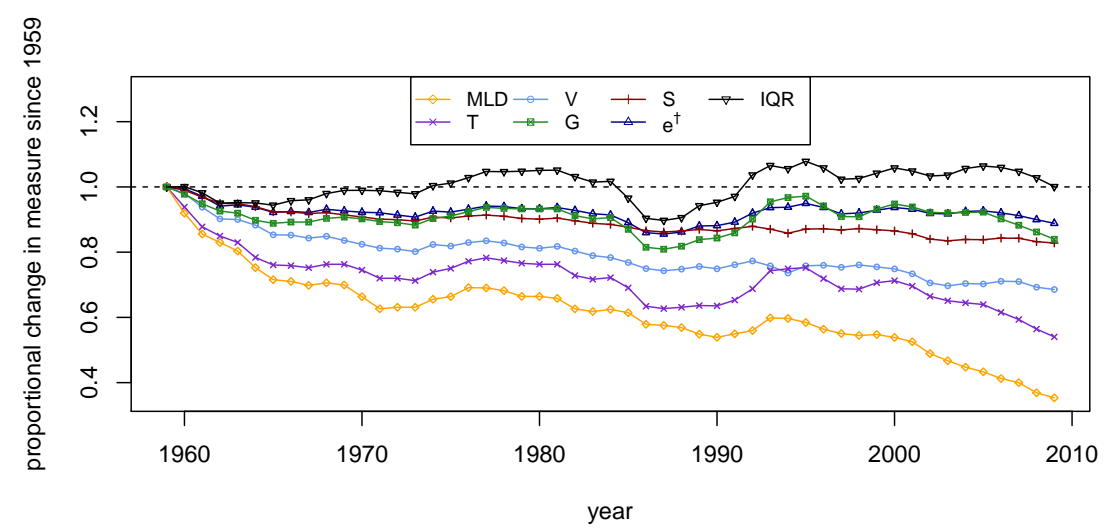

Figure 4: The indices of lifespan variability for Russian males, measured relative to their values in 1959. All indices were calculated from period lifetable data, 1959-2008, from the Human Mortality Database.

Figure 5 presents the LTRE decomposition of life expectancy and of the indices, relative to their starting value, computed using equation (17). Reductions in infant, child, and adolescent mortality led to gains in life expectancy, but increased mortality of adults age 20-70 tempered these gains. The positive contributions to life expectancy from reduced adult mortality during 1984-1987 are also visible.

The indices of variability show a different pattern. Their changes are a balance of strong negative contributions from infant and, to a lesser extent, child 


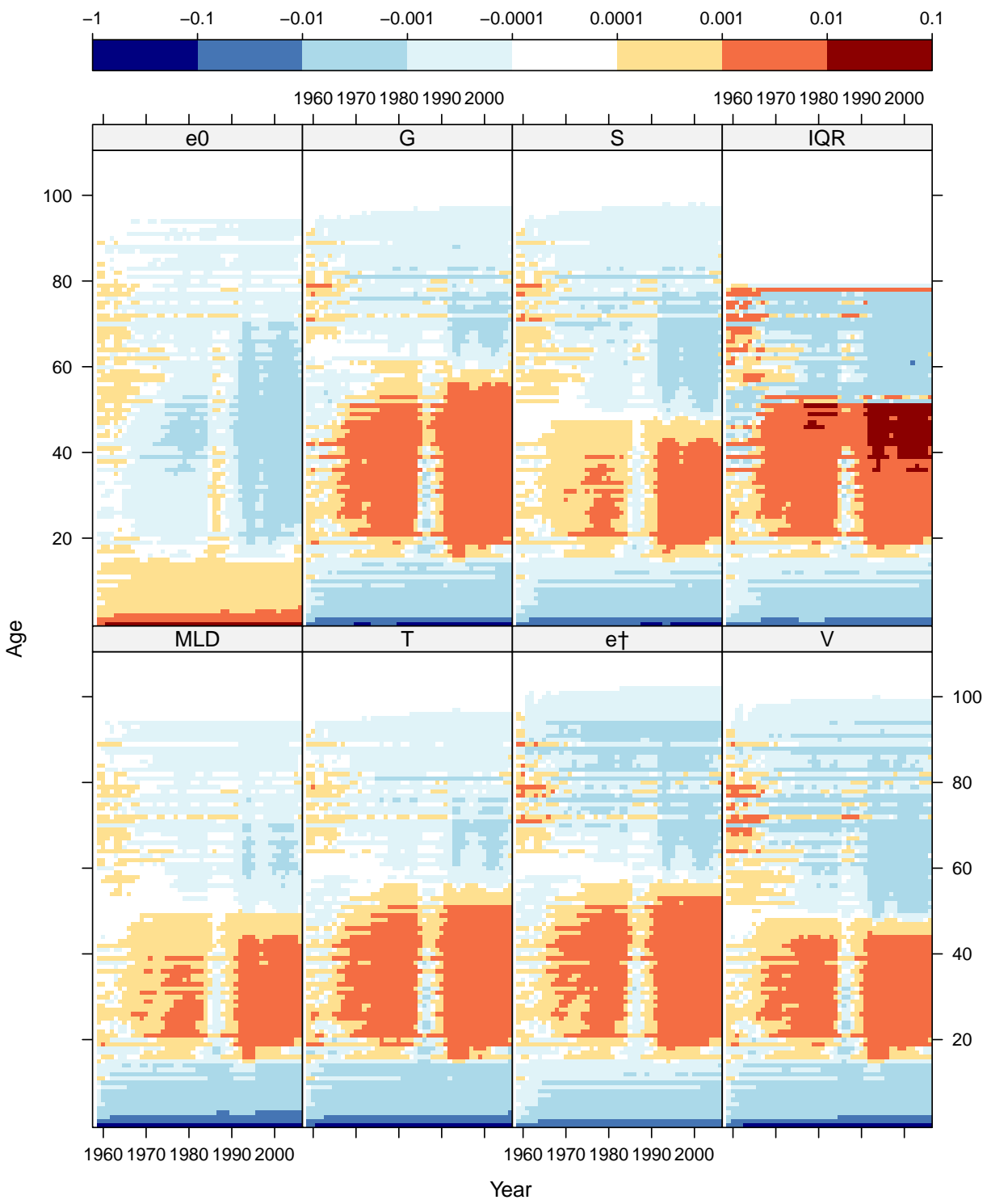

Figure 5: The proportional contribution of changes in age-specific mortality to the changes in life expectancy, $e_{0}$ and in each index, measured relative to their values in 1959. Values were calculated by integrating the LTRE contributions calculated from (15). Note that the color scale changes by a factor of 10, in order to make contributions from all ages visible on the graphs. Calculations were based on period lifetable data for Russian males, 1959-2008, from the Human Mortality Database. 
mortality, and positive contributions from changes in mortality in ages 20-50. Thus the change in variability (no matter how it is measured) is a balance of contributions from these two age ranges.

\section{Discussion}

\section{Summary of results}

Each of the seven indices of lifespan variation now posseses a directly computable sensitivity and elasticity to changes in age-specific mortality, derived from a consistent Markov chain formulation, with Matlab and $\mathrm{R}$ code supplied as an online appendix for calculation purposes. These formulae can be applied to any life table mortality schedule, period or cohort. The sensitivity patterns, applied to period data, showed similar broad patterns. Over time, the indices have become more sensitive to infant and child mortality, with $M L D, T$ and $V$ being especially sensitive. Although earlier mortality regimes produced large differences in the threshold age of the indices, this age was similar for all indices over recent mortality schedules.

\section{Indices of variability and distributive justice}

It is important to remember that the mere fact of variation in age at death does not imply heterogeneity of individuals or inequality in the conditions they experience. Indeed, in the extreme case in which every individual experienced identical age-constant mortality hazards, the standard deviation of longevity and the life disparity would both equal the life expectancy. This variation, due to individual stochasticity (Caswell 2009), greatly exceeds the variation in longevity observed in actual populations. However, although individual stochasticity leads to variation in ages at death, it is unlikely that the current distribution is owing entirely to individual stochasticity. If this were the case then we would not expect to find larger variation among lower socioeconomic groups (van Raalte et al. 2011) or large differences in variation between national populations with similar average mortality levels (Edwards and Tuljapurkar 2005; Smits and Monden 2009; Vaupel et al. 2011). Danish twin studies have estimated that genetic processes account for about a quar- 
ter of the the total variation in lifespan (Herskind et al. 1996; McGue et al. 1993). Although substantial, this still leaves a large unexplained component.

To the extent that variation does represent the result of inequality in conditions, it may be interesting to think of it in terms of concepts of distributive justice. Distributive justice is concerned with how a society or group should allocate its scarce resources or product among individuals with competing needs or claims (Roemer 1996). Different notions of inequality arise from two different types of diversity: the basic individual heterogeneity and the assessment of inequality in terms of different variables (Sen 1992).

Most distributive justice frameworks can broadly fit into four categories: pro-poor, egalitarian, utilitarian and minimum threshold frameworks (Marchand, Wikler and Landesman 1998). An interesting line of future research would be to view changes in the age-at-death distribution through the lens of distributive justice. To this extent, perturbation analysis could be used to guide the selection of an index depending on how the sensitivity results align with the aimed-for distributive justice approach. Such delineations are dependent on the particular mortality schedule. As can be seen in Figure 2, the interquartile range was most sensitive to infant mortality in the French male 1918 distribution, but least sensitive in 1948 and 2008.

Once a definition of inequality (and corresponding index) is chosen, perturbation results could further be used to target policy interventions to ages where reductions in mortality best reduce inequality.

\section{Conclusion}

We compared seven indices of lifespan variation, all of which largely correlated with one another over the mortality schedules found in the 7516 unique lifetables of the Human Mortality Database. Using matrix differentiation techniques we derived the expressions for the sensitivities and elasticities of all indices. We highlighted four key uses of these perturbation results: (1) quantifying the effect of an age-specific mortality intervention on lifespan variation, (2) analytically determining the threshold age separating deaths at ages which increase or decrease variation in ages at death, (3) providing the output for a LTRE decomposition analysis that quantifies the changes in any lifespan variation index into contributions from mortality change at each 
age, and (4) comparing indices on the basis of their sensitivities to changes in age-specific mortality.

\section{Acknowledgements}

AvR is supported by the Max Planck Society. HC acknowledges financial support from the U.S. National Science Foundation (DEB-0816514 and SES1156378), the Woods Hole Oceanographic Institution, and a Research Award from the Alexander von Humboldt Foundation, and the hospitality of the Max Planck Institute for Demographic Research. The International Max Planck Research School of Demography provided support for a course (IMPRSD 133) in which some of these ideas were first explored. The authors would additionally like to thank Jim Vaupel, Johan Mackenbach, Mikko Myrskyl, and two anonymous reviewers for helpful comments. An earlier version of this paper was presented at the annual meeting of the Population Association of America in San Francisco, CA. 


\section{Appendix - Sensitivity Results and Deriva- tions}

This appendix provides details of the derivations. We begin with a summary of some basic matrix calculus techniques, and then present the derivations of the sensitivities of the indices of lifespan variation.

\subsection{Matrix calculus preliminaries}

The indices of lifespan variation in Table 3 are functions of scalars, vectors and matrices. Matrix calculus permits differentiation of all three. The derivative of a scalar $y$ with respect to a scalar $x$ is the derivative $\frac{d y}{d x}$ familiar from basic calculus. The derivative of a $n \times 1$ vector $\mathbf{y}$ with respect to a scalar $x$ is the $n \times 1$ vector

$$
\frac{d \mathbf{y}}{d x}=\left(\begin{array}{c}
\frac{d y_{1}}{d x} \\
\vdots \\
\frac{d y_{n}}{d x}
\end{array}\right)
$$

The derivative of a scalar $y$ with respect to a $m \times 1$ vector $\mathbf{x}$ is the $1 \times m$ gradient vector

$$
\frac{d y}{d \mathbf{x}^{\top}}=\left(\frac{\partial y}{d x_{1}} \cdots \frac{\partial y}{d x_{m}}\right) .
$$

The derivative of an $n \times 1$ vector $\mathbf{y}$ with respect to a $m \times 1$ vector $\mathbf{x}$ is the $n \times m$ Jacobian matrix, whose $(i, j)$ entry is the derivative of $y_{i}$ with respect to $x_{j}$ :

$$
\frac{d \mathbf{y}}{d \mathbf{x}^{\top}}=\left(\frac{d y_{i}}{d x_{j}}\right)
$$

The derivatives of matrices are computed by transforming the matrices into column vectors using the vec operator and applying the rules for vector differentiation. Thus the derivative of the $m \times n$ matrix $\mathbf{Y}$ with respect to the $p \times q$ matrix $\mathbf{X}$ is the $m n \times p q$ matrix

$$
\frac{d \mathbf{Y}}{d \mathbf{X}}=\frac{d \operatorname{vec} \mathbf{Y}}{d \operatorname{vec}^{\top} \mathbf{X}} .
$$

For notational simplicity we denote $(d \mathrm{vec} \mathbf{X})^{\top}$ as $d \operatorname{vec}^{\top} \mathbf{X}$. 
These definitions imply the chain rule for matrix calculus; if $\mathbf{Y}$ is a function of $\mathbf{X}$, and $\mathbf{X}$ is a function of $\mathbf{Z}$, then

$$
\frac{d \operatorname{vec} \mathbf{Y}}{d \operatorname{vec}^{\top} \mathbf{Z}}=\frac{d \operatorname{vec} \mathbf{Y}}{d \operatorname{vec}^{\top} \mathbf{X}} \frac{d \operatorname{vec} \mathbf{X}}{d \operatorname{vec}^{\top} \mathbf{Z}} .
$$

Matrix derivatives are constructed by forming differentials, where the differential of a matrix (or vector) is the matrix (or vector) of differentials of the elements; i.e.

$$
d \mathbf{X}=\left(d x_{i j}\right)
$$

If, for some matrix $\mathbf{Q}$, it can be shown that

$$
d \mathbf{y}=\mathbf{Q} d \mathbf{x}
$$

then according to the "first identification theorem" of Magnus and Neudecker (Magnus and Neudecker 1985)

$$
\frac{d \mathbf{y}}{d \mathbf{x}^{\top}}=\mathbf{Q}
$$

We will frequently obtain expressions of the form (25) using a theorem originally due to Roth (Roth 1934), that if $\mathbf{Y}=\mathbf{A B C}$ then

$$
\operatorname{vec} \mathbf{Y}=\left(\mathbf{C}^{\top} \otimes \mathbf{A}\right) \operatorname{vec} \mathbf{B} .
$$

We will also simplify expressions involving Kronecker products using

$$
(\mathbf{A} \otimes \mathbf{B})(\mathbf{C} \otimes \mathbf{D})=\mathbf{A C} \otimes \mathbf{B D} .
$$

whenever AC and BD are defined.

More details on matrix calculus can be found in Magnus and Neudecker (1988). A good mathematical introduction is in Abadir and Magnus (2005), and demographic discussions appear in Caswell (2007; 2008; 2010).

\subsection{Sensitivities of the indices of lifespan variation}

\subsubsection{Preliminaries}

Differentiating the various indices made use of the following sensitivities. The vector of life expectancies as a function of age is given by

$$
\boldsymbol{\eta}^{\top}=\mathbf{e}^{\top} \mathbf{N}
$$


The derivative of this vector with respect to mortality is (Caswell 2006; 2009)

$$
\frac{d \boldsymbol{\eta}}{d \boldsymbol{\theta}^{\top}}=\left(\mathbf{I} \otimes \mathbf{e}^{\top}\right)\left(\mathbf{N}^{\top} \otimes \mathbf{N}\right) \frac{d \mathrm{vec} \mathbf{U}}{d \boldsymbol{\theta}^{\top}} .
$$

The life expectancy at birth is given by

$$
\eta_{1}=\boldsymbol{\eta}^{\top} \mathbf{e}_{1}
$$

and thus its derivative is

$$
\begin{aligned}
\frac{d \eta_{1}}{d \boldsymbol{\theta}^{\top}} & =\left(\mathbf{e}_{1}^{\top} \otimes \mathbf{e}^{\top}\right) \frac{d \operatorname{vec} \mathbf{N}}{d \boldsymbol{\theta}^{\top}} \\
& =\left(\mathbf{e}_{1}^{\top} \mathbf{N}^{\top} \otimes \mathbf{e}^{\top} \mathbf{N}\right) \frac{d \operatorname{vec} \mathbf{U}}{d \boldsymbol{\theta}^{\top}}
\end{aligned}
$$

The distribution of age at death is given by the vector

$$
\mathbf{f}=\mathrm{MNe}_{1}
$$

Its derivative is given by (Caswell 2010),

$$
\frac{d \mathbf{f}}{d \boldsymbol{\theta}^{\top}}=\left(\mathbf{e}_{1}^{\top} \mathbf{N}^{\top} \otimes \mathbf{I}\right) \frac{d \mathrm{vec} \mathbf{M}}{d \boldsymbol{\theta}^{\top}}+\left(\mathbf{e}_{1}^{\top} \mathbf{N}^{\top} \otimes \mathbf{B}\right) \frac{d \operatorname{vec} \mathbf{U}}{d \boldsymbol{\theta}^{\top}}
$$

The derivatives of $\mathbf{U}$ and $\mathbf{M}$ depend on the structure of the life cycle; in the ageclassified case under consideration here, $\mathbf{M}$ contains the probabilities of death $q_{i}$ on the diagonal, and $\mathbf{U}$ contains the probabilities of survival $1-q_{i}$ on the subdiagonal.

\subsubsection{Life disparity $\eta^{\dagger}$}

The disparity can be written

$$
\eta^{\dagger}=\mathbf{f}^{\top} \boldsymbol{\eta}
$$

As shown in Caswell $(2010,2011)$,

$$
d \eta^{\top}=\left(d \mathbf{f}^{\top}\right) \boldsymbol{\eta}+\mathbf{f}^{\top}(d \boldsymbol{\eta})
$$

and thus

$$
\frac{d \eta^{\dagger}}{d \boldsymbol{\theta}^{\top}}=\boldsymbol{\eta}^{\top} \frac{d \mathbf{f}}{d \boldsymbol{\theta}^{\top}}+\mathbf{f}^{\top} \frac{d \boldsymbol{\eta}}{d \boldsymbol{\theta}^{\top}}
$$

where $d \mathbf{f} / d \boldsymbol{\theta}^{\top}$ is given by (34) and $d \boldsymbol{\eta} / d \boldsymbol{\theta}^{\top}$ is given by (29). 


\subsubsection{Gini coefficient}

In matrix form, the Gini coefficient is given by

$$
G=1-\frac{1}{\eta_{1}} \mathbf{e}^{\top}[\ell \circ \ell]
$$

where the survivorship vector is

$$
\ell=\mathbf{e}-\mathbf{C f}
$$

Differentiating (38), noting that $\eta_{1}$ is a scalar, gives

$$
d G=\frac{1}{\eta_{1}^{2}} \mathbf{e}^{\top}(\boldsymbol{\ell} \circ \boldsymbol{\ell}) d \eta_{1}-\frac{2}{\eta_{1}} \mathbf{e}^{\top}[\boldsymbol{\ell} \circ(d \boldsymbol{\ell})]
$$

We apply the vec operator to both sides of (40) and obtain

$$
d G=\frac{1}{\eta_{1}^{2}} \mathbf{e}^{\top}(\boldsymbol{\ell} \circ \boldsymbol{\ell}) d \eta_{1}-\frac{2}{\eta_{1}} \ell^{\top} d \boldsymbol{\ell}
$$

Differentiating (39) gives $d \boldsymbol{\ell}=-\mathbf{C} d \mathbf{f}$; substituting this into (41) and using the chain rule gives

$$
\frac{d G}{d \boldsymbol{\theta}^{\top}}=\frac{1}{\eta_{1}^{2}} \mathbf{e}^{\top}(\boldsymbol{\ell} \circ \boldsymbol{\ell}) \frac{d \eta_{1}}{d \boldsymbol{\theta}^{\top}}+\frac{2}{\eta_{1}} \ell^{\top} \mathbf{C} \frac{d \mathbf{f}}{d \boldsymbol{\theta}^{\top}}
$$

where $d \eta_{1} / d \boldsymbol{\theta}^{\top}$ is given by $(32)$

\subsubsection{Mean Logarithmic Deviation}

The mean logarithmic deviation in matrix notation is

$$
M L D=\mathbf{f}^{\top}\left[\mathbf{e} \log \eta_{1}-\log \mathbf{x}\right]
$$

where the logarithm is applied elementwise. Differentiating (43) gives

$$
d M L D=\left(d \mathbf{f}^{\top}\right)\left[\mathbf{e} \log \eta_{1}-\log \mathbf{x}\right]+\mathbf{f}^{\top} \mathbf{e}\left(d \log \eta_{1}\right)
$$

However, $\mathbf{f}^{\top} \mathbf{e}=1$ because $\mathbf{f}$ is a probability distribution. Using this fact and also noting that $d \log \eta_{1}=\left(1 / \eta_{1}\right) d \eta_{1}$, we obtain

$$
\frac{d M L D}{d \boldsymbol{\theta}^{\top}}=\left[\mathbf{e}^{\boldsymbol{\top}} \log \eta_{1}-\log \mathbf{x}^{\boldsymbol{\top}}\right] \frac{d \mathbf{f}}{d \boldsymbol{\theta}^{\top}}+\frac{1}{\eta_{1}} \frac{d \eta_{1}}{d \boldsymbol{\theta}^{\boldsymbol{\top}}} .
$$

where $d \eta_{1} / d \boldsymbol{\theta}^{\top}$ is given by (32) and $d \mathbf{f} / d \boldsymbol{\theta}^{\top}$ is given by (34). 


\subsubsection{Theil's index}

The expression for Theil's index in matrix notation is

$$
T=\mathbf{f}^{\top}\left[\frac{\mathbf{x}}{\eta_{1}} \circ \log \frac{\mathbf{x}}{\eta 1}\right]
$$

where the logarithm is applied elementwise. Differentiating (46) term by term yields

$$
d T=\left(d \mathbf{f}^{\top}\right)\left[\frac{\mathbf{x}}{\eta_{1}} \circ \log \frac{\mathbf{x}}{\eta 1}\right]+\mathbf{f}^{\top}\left[d\left(\frac{\mathbf{x}}{\eta_{1}}\right) \circ \log \frac{\mathbf{x}}{\eta_{1}}\right]+\mathbf{f}^{\top}\left[\frac{\mathbf{x}}{\eta_{1}} \circ d\left(\log \frac{\mathbf{x}}{\eta_{1}}\right)\right] .
$$

However,

$$
\begin{aligned}
d\left(\frac{\mathbf{x}}{\eta_{1}}\right) & =-\frac{\mathbf{x}}{\eta_{1}^{2}} d \eta_{1} \\
d\left(\log \frac{\mathbf{x}}{\eta_{1}}\right) & =d\left(\log \mathbf{x}-\mathbf{e} \log \eta_{1}\right) \\
& =-\frac{\mathbf{e}}{\eta_{1}} d \eta_{1}
\end{aligned}
$$

Substituting (48) and (49) into (47), and transposing the first term, gives

$$
d T=\left(\frac{\mathbf{x}^{\top}}{\eta_{1}} \circ \log \frac{\mathbf{x}^{\top}}{\eta_{1}}\right) d \mathbf{f}-\mathbf{f}^{\top}\left[\left(\frac{\mathbf{x}}{\eta_{1}^{2}} \circ \log \frac{\mathbf{x}}{\eta_{1}}\right)+\left(\frac{\mathbf{x}}{\eta_{1}} \circ \frac{\mathbf{e}}{\eta_{1}}\right)\right] d \eta_{1}
$$

Simplifying equation (50) and expressing the result in terms of a parameter vector $\boldsymbol{\theta}$ gives

$$
\frac{d T}{d \boldsymbol{\theta}^{\top}}=\left(\frac{\mathbf{x}^{\top}}{\eta_{1}} \circ \log \frac{\mathbf{x}^{\top}}{\eta_{1}}\right) \frac{d \mathbf{f}}{d \boldsymbol{\theta}^{\top}}-\left(\frac{T}{\eta_{1}}+\frac{\mathbf{f}^{\top} \mathbf{x}}{\eta_{1}^{2}}\right) \frac{d \eta_{1}}{d \boldsymbol{\theta}^{\top}}
$$

where $d \eta_{1} / d \boldsymbol{\theta}^{\top}$ is given by (32) and $d \mathbf{f} / d \boldsymbol{\theta}^{\top}$ is given by (34).

\subsubsection{The variance and standard deviation of longevity}

The variance in longevity, conditional upon survival to age class $i$, is given by the vector $\mathbf{v}$, which satisfies

$$
\mathbf{v}^{\top}=\mathbf{e}^{\top} \mathbf{N}(2 \mathbf{N}-\mathbf{I})-\boldsymbol{\eta}^{\top} \circ \boldsymbol{\eta}^{\top}
$$

Caswell $(2006,2009,2010)$ shows that

$$
\frac{d \mathbf{v}}{d \boldsymbol{\theta}^{\top}}=\left[2\left(\mathbf{N}^{\top} \otimes \mathbf{e}^{\boldsymbol{\top}}\right)+2\left(\mathbf{I} \otimes \mathbf{e}^{\top} \mathbf{N}\right)-\left(\mathbf{I} \otimes \mathbf{e}^{\top}\right)\right] \frac{d \mathrm{vec} \mathbf{N}}{d \boldsymbol{\theta}^{\top}}-2 \operatorname{diag}(\boldsymbol{\eta}) \frac{d \boldsymbol{\eta}}{d \boldsymbol{\theta}^{\top}}
$$


where $d \boldsymbol{\eta} / d \boldsymbol{\theta}^{\top}$ is given by (29) and

$$
\frac{d \operatorname{vec} \mathbf{N}}{d \boldsymbol{\theta}^{\top}}=\left(\mathbf{N}^{\top} \otimes \mathbf{N}\right) \frac{d \operatorname{vec} \mathbf{U}}{d \boldsymbol{\theta}^{\top}} .
$$

The standard deviation of longevity is given by the vector

$$
\mathbf{s}=\sqrt{\mathbf{v}}
$$

where the square root is taken elementwise, and its sensitivity was derived in Caswell (2010),

$$
\frac{d \mathbf{s}}{d \boldsymbol{\theta}^{\top}}=\frac{1}{2} \operatorname{diag}(\mathbf{s})^{-1} \frac{d \mathbf{v}}{d \boldsymbol{\theta}^{\top}} .
$$

\subsubsection{The inter-quartile range}

The inter-quartile range is defined implicitly in terms of the distribution of ages

at death. Let $f(x)$ be a probability density function and $F(x)=\int_{-\infty}^{x} f(s) d s$ be the cumulative distribution. The $q$ th quantile is the value $\hat{x}$ satisfying

$$
F(\hat{x})=q
$$

Let $F\left(\hat{x}_{1}\right)=q_{1}$ and $F\left(\hat{x}_{2}\right)=q_{2}$, assuming that $q_{2}>q_{1}$. The inter-quantile range is

$$
R\left(q_{1}, q_{2}\right)=\hat{x_{2}}-\hat{x_{1}}
$$

The special case of the inter-quartile range refers to $R(0.25,0.75)$.

Now we choose a set of probabilities of interest

$$
\mathbf{q}=\left(\begin{array}{c}
q_{1} \\
\vdots \\
q_{h}
\end{array}\right)
$$

and let $\hat{\mathbf{x}}$ be the vector of quantiles that satisfy

$$
F[\boldsymbol{\theta}, \hat{\mathbf{x}}(\boldsymbol{\theta})]=\mathbf{q},
$$

where the distribution $f(\cdot)$ depends on a parameter vector $\boldsymbol{\theta}$, of dimension $p \times 1$. Next we differentiate equation (60)

$$
\frac{\partial F}{\partial \boldsymbol{\theta}^{\top}} d \boldsymbol{\theta}+\frac{\partial F}{\partial \hat{\mathbf{x}}^{\top}} d \hat{\mathbf{x}}=0
$$


and solve for $d \hat{\mathbf{x}}$, to obtain

$$
d \hat{\mathbf{x}}=-\left(\frac{\partial F}{\partial \hat{\mathbf{x}}^{\top}}\right)^{-1}\left(\frac{\partial F}{\partial \boldsymbol{\theta}^{\top}}\right) d \boldsymbol{\theta} .
$$

The first identification theorem implies that

$$
\frac{d \hat{\mathbf{x}}}{d \boldsymbol{\theta}^{\top}}=-\left(\frac{\partial F}{\partial \hat{\mathbf{x}}^{\top}}\right)^{-1}\left(\frac{\partial F}{\partial \boldsymbol{\theta}^{\top}}\right)
$$

The first term on the right hand side of of equation (63) is

$$
\left(\frac{\partial F}{\partial \hat{\mathbf{x}}^{\top}}\right)^{-1}=\left(\begin{array}{ccc}
\frac{1}{f\left(\hat{x}_{1}\right)} & & 0 \\
& \ddots & \\
0 & & \frac{1}{f\left(\hat{x}_{h}\right)}
\end{array}\right)
$$

while the second term is

$$
\left(\frac{\partial F}{\partial \theta^{\top}}\right)=\left(\begin{array}{ccc}
\frac{\partial F\left(\hat{x}_{1}\right)}{\partial \theta_{1}} & \cdots & \frac{\partial F\left(\hat{x}_{1}\right)}{\partial \theta_{p}} \\
\vdots & & \vdots \\
\frac{\partial F\left(\hat{x}_{h}\right)}{\partial \theta_{1}} & \cdots & \frac{\partial F\left(\hat{x}_{h}\right)}{\partial \theta_{p}}
\end{array}\right)
$$

The product of equations (64) and (65, following equation (63) gives

$$
\left(\frac{d \hat{x}}{d \theta^{\top}}\right)=-\left(\begin{array}{ccc}
\frac{1}{f\left(\hat{x}_{1}\right)} \frac{\partial F\left(\hat{x}_{1}\right)}{\partial \theta_{1}} & \cdots & \frac{1}{f\left(\hat{x}_{1}\right)} \frac{\partial F\left(\hat{x}_{1}\right)}{\partial \theta_{p}} \\
\vdots & & \vdots \\
\frac{1}{f\left(\hat{x}_{h}\right)} \frac{\partial F\left(\hat{x}_{h}\right)}{\partial \theta_{1}} & \cdots & \frac{1}{f\left(\hat{x}_{h}\right)} \frac{\partial F\left(\hat{x}_{h}\right)}{\partial \theta_{p}}
\end{array}\right)
$$

The sensitivity of the inter-quantile range is the difference between row $j$ and row $i$ of (66).

$$
\frac{d R_{(i, j)}}{d \boldsymbol{\theta}^{\top}}=\frac{d \hat{x}_{j}}{d \boldsymbol{\theta}^{\top}}-\frac{d \hat{x}_{i}}{d \boldsymbol{\theta}^{\top}}
$$

When $\mathbf{f}(x)$ is a discrete distribution, the quantiles will have to be interpolated. This is what we did to find the sensitivity of the $I Q R$ with quartiles $\hat{x}_{3}$ and $\hat{x}_{1}$. 


\section{Appendix - Matlab code}

The perturbation analysis results here are obtained using matrix operations, and optimally computed using software designed for matrix calculations. The best such software is MATLAB, which is a nearly universal standard in physics, engineering, and mathematics. The widely used statistical package $\mathrm{R}$ can also perform the calculations, although it is not primarily a matrix language. In this section we provide MATLAB code, and in the next we provide the corresponding $\mathrm{R}$ code, for the calculations.

\subsection{Function needed in your working directory, titled $\mathrm{vec} \cdot \mathrm{m}$ \\ function $\mathrm{v}=\operatorname{vec}(\mathrm{x})$ \\ $\mathrm{V}=\mathrm{x}(:) ;$}

\subsection{Main code}

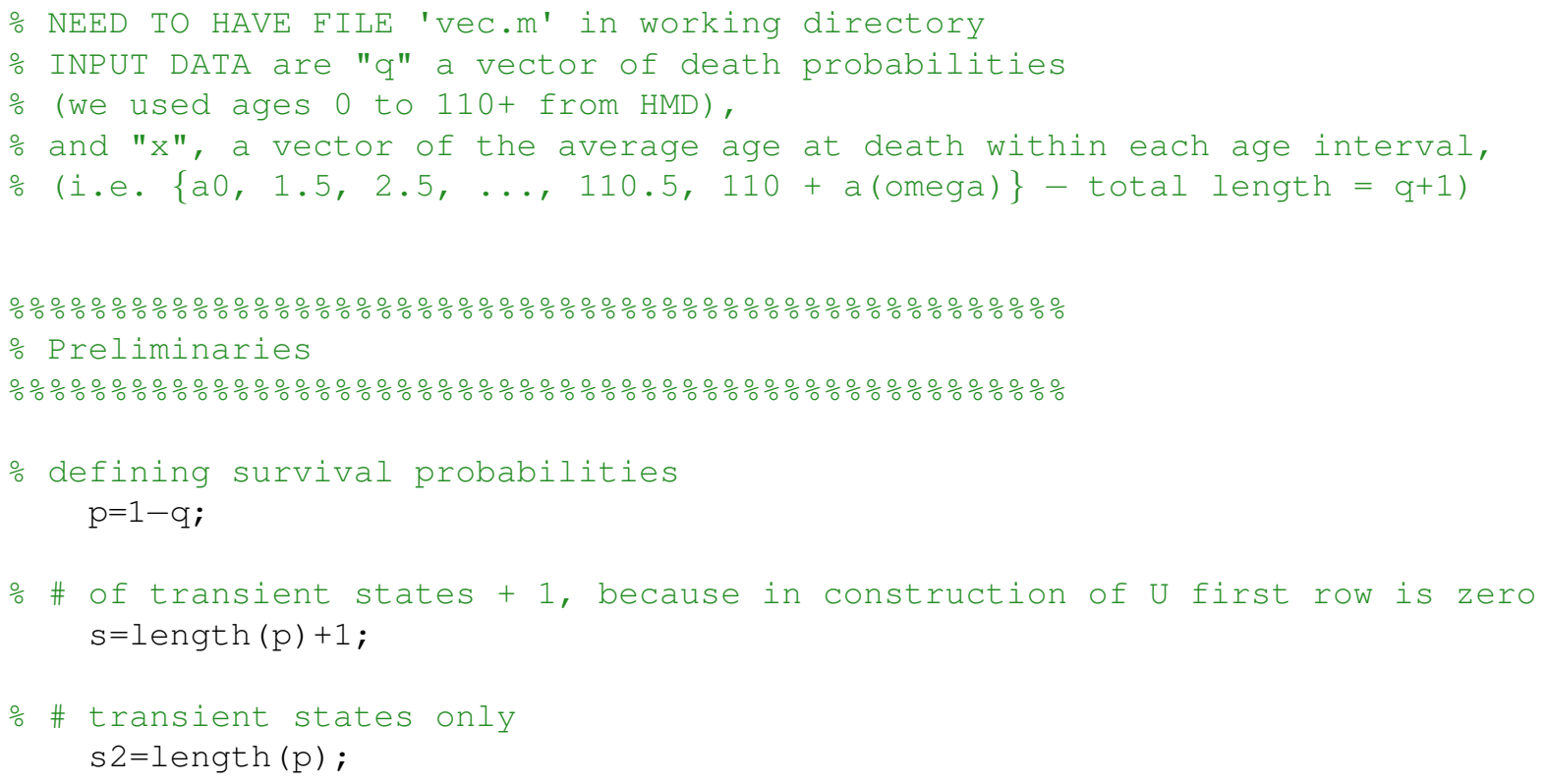




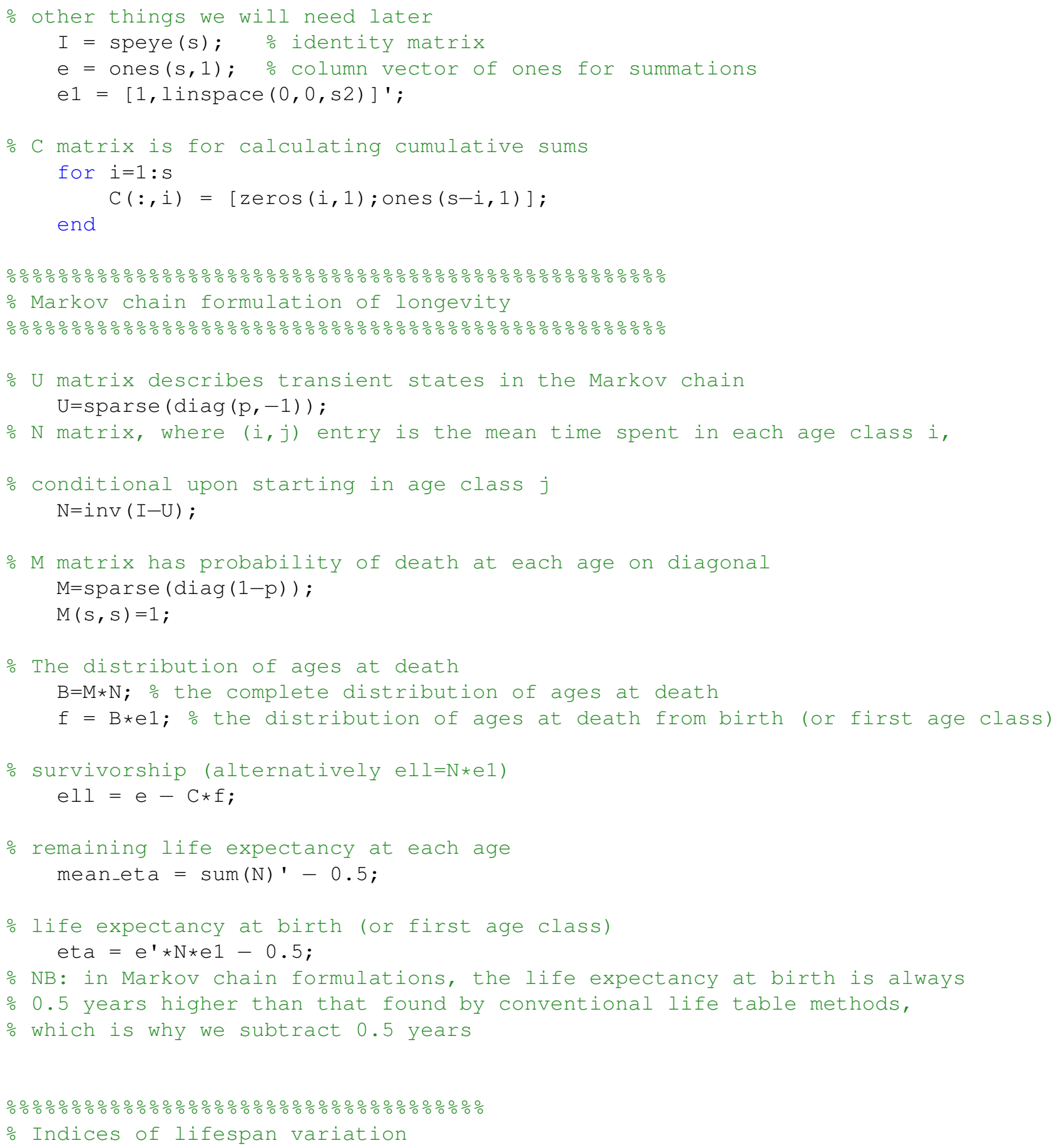




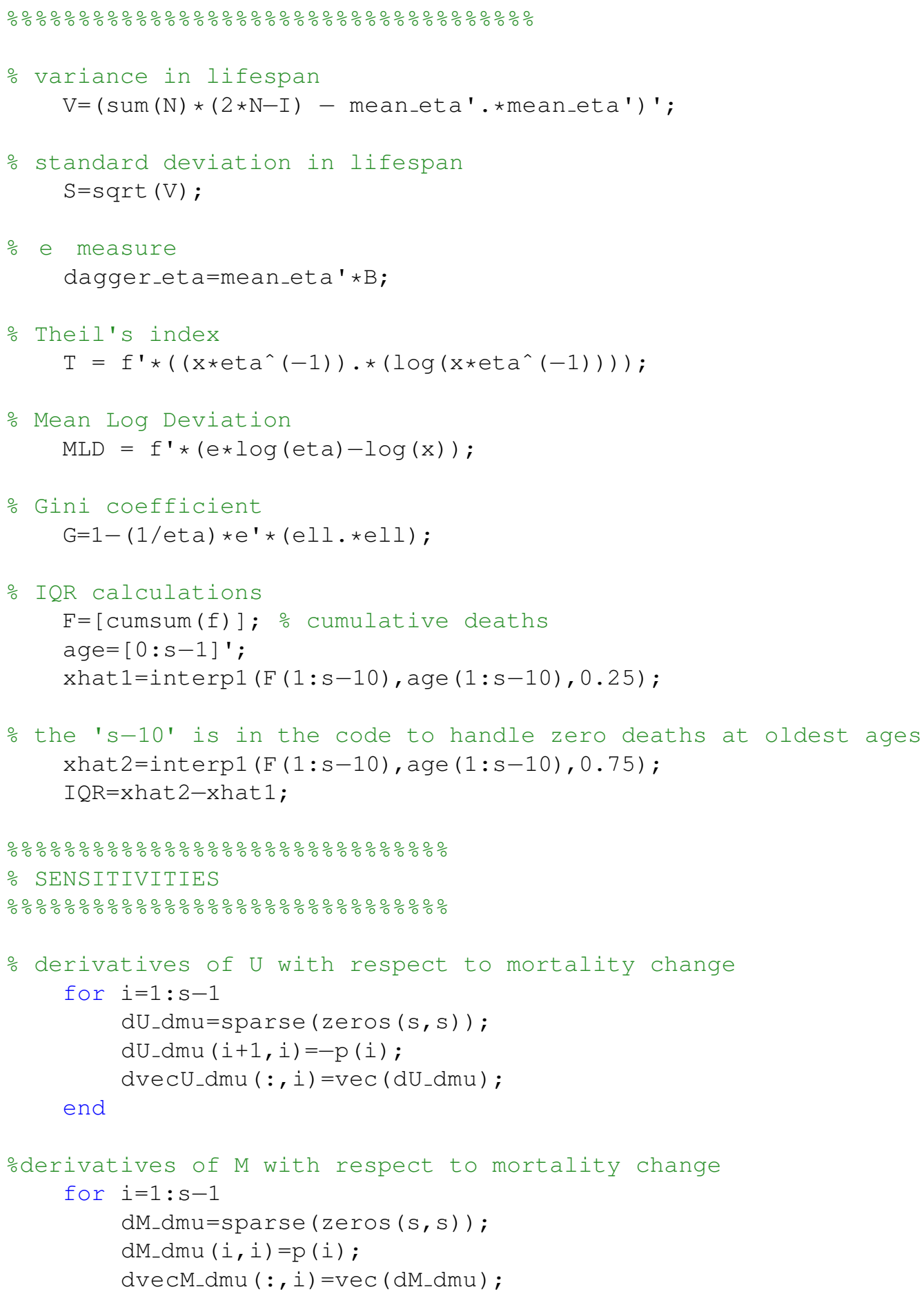


end

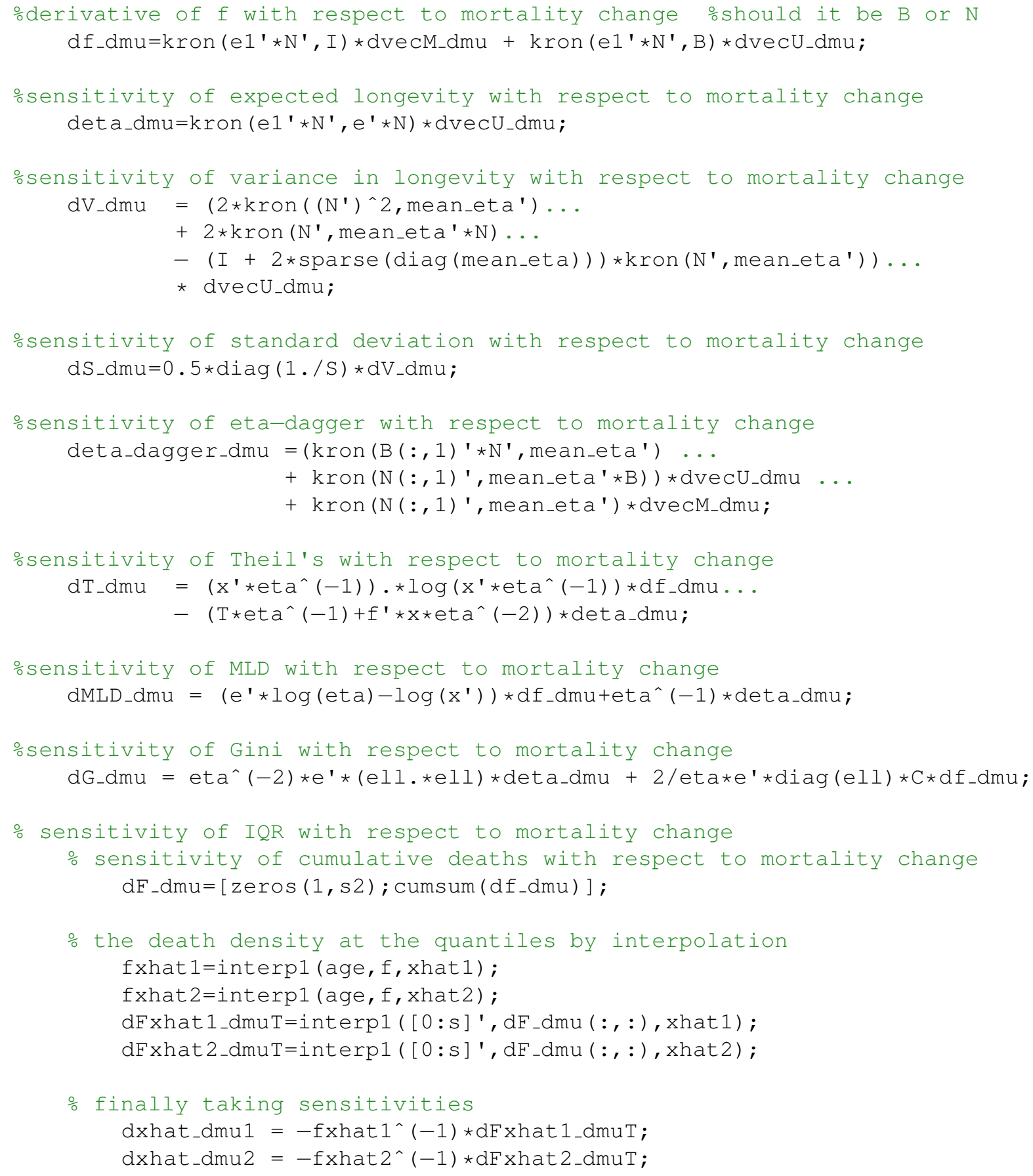


dIQR_dmu=dxhat_dmu2-dxhat_dmu1;

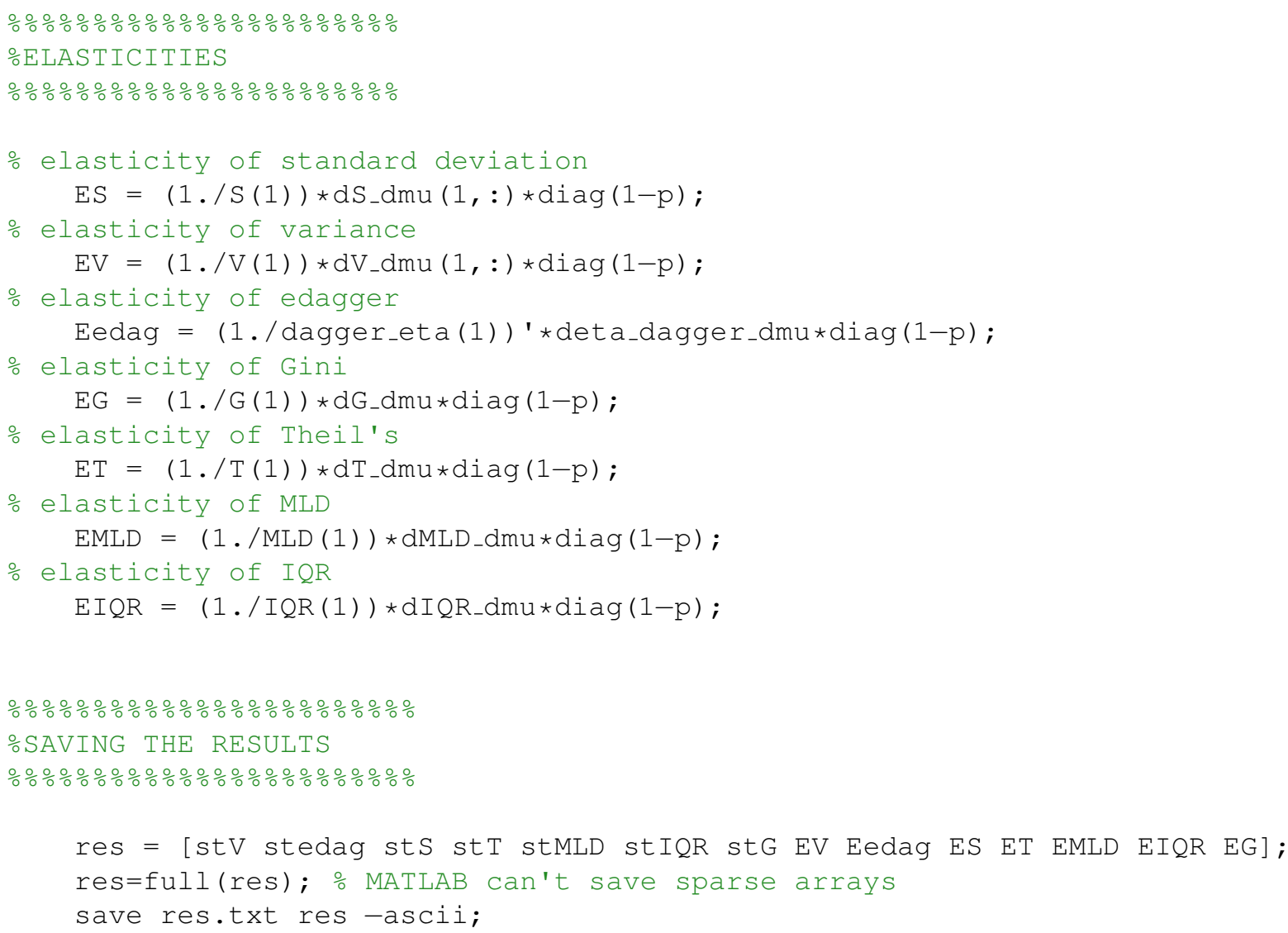




\section{Appendix - $\mathrm{R}$ code}

\# R code for paper "Perturbation analysis of indices of lifespan variability"

\# This code was designed for readability, not efficiency

$\operatorname{rm}($ list $=1 \mathrm{~s}(\mathrm{all}=\mathrm{TRUE}))$

options (scipen=6)

\#----------------------------
\#------ Input data

\#-- "q" a vector of death probabilities (we used ages 0 to $110+$ from HMD), \#-- and " $x$ ", a vector of the average age at death within each age interval \#-- (i.e. a0, 1.5, 2.5 etc., length qi+1)

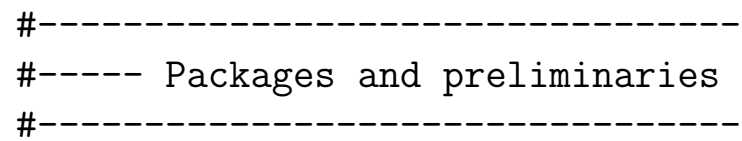

library(Matrix) \# for sparse matrices

library(signal) \# for interp1 function

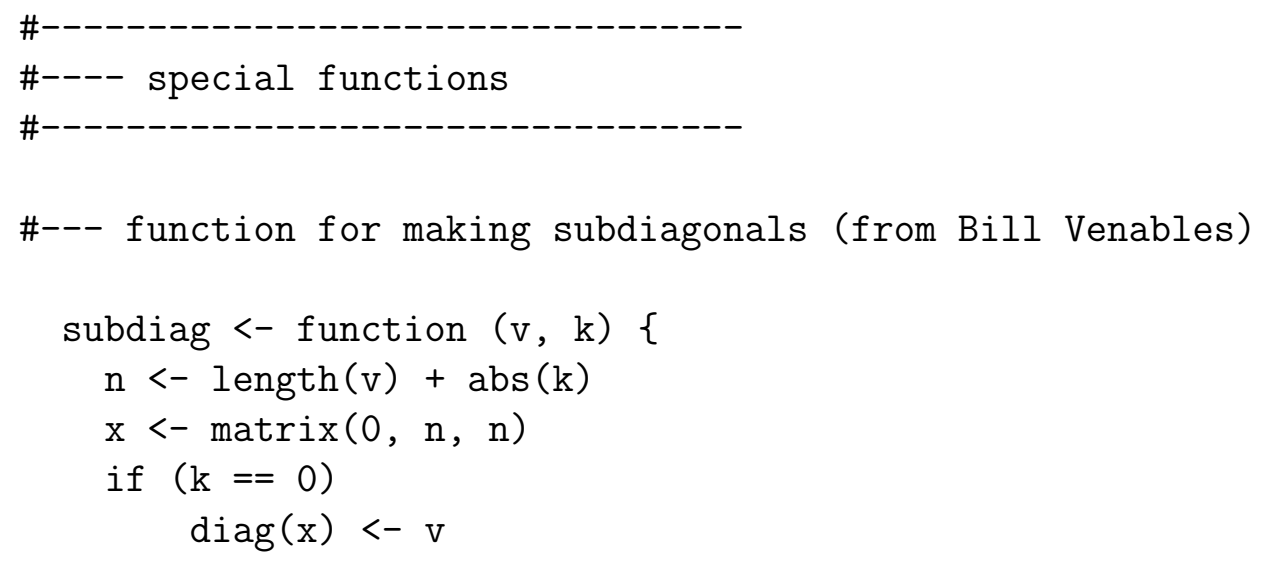


\# Markov chain formulation of longevity

\#---------------------------------------------

\# U matrix describes transient states in the Markov chain

$\mathrm{U}<-\operatorname{subdiag}(\mathrm{p},-1)$

$\mathrm{U}<-$ as (U, "sparseMatrix")

\# $N$ matrix, where $(i, j)$ entry is the mean time spent in each age class $i$,

\# conditional upon starting in age class $j$

$\mathrm{N}<-$ solve $(\mathrm{I}-\mathrm{U})$

$\mathrm{N}<-\operatorname{as}(\mathrm{N}$, "sparseMatrix")

\# M matrix has probability of death at each age on diagonal

$M<-\operatorname{diag}(c(q, 1))$

M $<-$ as $(M$, "sparseMatrix")

\# The distribution of ages at death

$\mathrm{B}<-\mathrm{M} \% * \% \mathrm{~N}$ \# the complete distribution of ages at death

$f<-B \% * \%$ e1 \# the distribution of ages at death from birth (1st age class)

B $<-$ as (B, "sparseMatrix")

\# survivorship (alternatively ell <- e-C \% $* \%$ )

ell $<-\mathrm{N} \% * \%$ e1

ell <- as (ell, "sparseMatrix")

\# remaining life expectancy at each age

mean_eta $<-\operatorname{colSums}(\mathrm{N})-0.5$

\# life expectancy at birth (or first age class)

eta $<-$ mean_eta[1]

\# NB: in Markov chain formulations, the life expectancy at birth is always

\# 0.5 years higher than that found by conventional life table methods, \# which is why we subtract 0.5 years

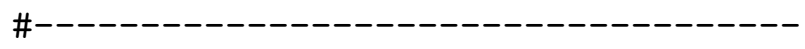




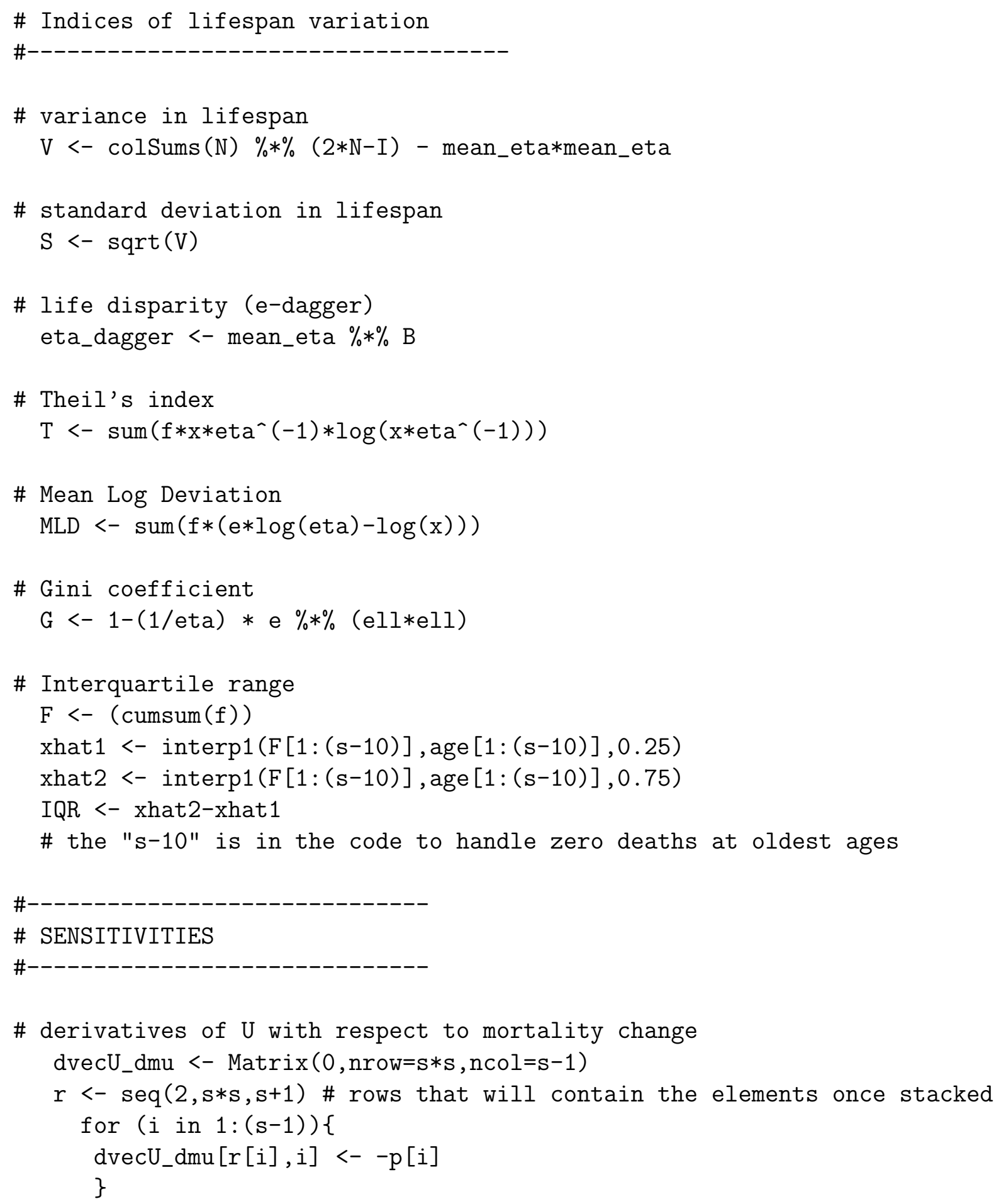


dvecU_dmu <- as (dvecU_dmu, "sparseMatrix")

\# derivatives of $\mathrm{M}$ with respect to mortality change

dvecM_dmu <- Matrix $(0$, nrow=s*s, ncol=s -1$)$

r2 <- seq(1,s*s,s+1) \# rows that will contain the elements once stacked for (i in 1: $(s-1))\{$

dvecM_dmu[r2[i],i] <- $p[i]$

\}

dvecM_dmu <- as (dvecM_dmu, "sparseMatrix")

\# derivative of $f$ with respect to mortality change df_dmu <- $t(\operatorname{kronecker}(\mathrm{N}[, 1], \mathrm{I})) \% * \%$ dvecM_dmu + $\mathrm{t}(\operatorname{kronecker}(\mathrm{N}[, 1], \mathrm{t}(\mathrm{B}))) \% * \%$ dvecU_dmu

\# sensitivity of expected longevity with respect to mortality change deta_dmu <- $t(\operatorname{kronecker}(\mathrm{N}[, 1], \operatorname{col} \operatorname{Sums}(\mathrm{N}))) \%$ dvecU_dmu

\# sensitivity of variance in longevity with respect to mortality change $\mathrm{dV}_{-} \mathrm{dmu}<-(2 * \operatorname{kronecker}(\mathrm{t}(\mathrm{N}) \% * \% \mathrm{t}(\mathrm{N}), \mathrm{t}($ mean_eta $))+$

$2 *$ kronecker $(t(N)$, mean_eta $\% * \% N)$ -

$(I+2 *($ diag $($ mean_eta $))) \% * \% \operatorname{kronecker}(\mathrm{t}(\mathrm{N}), \mathrm{t}($ mean_eta $))) \% * \%$ dvecU_dmu

\# sensitivity of standard deviation with respect to mortality change dS_dmu <- $0.5 * \operatorname{diag}($ as.vector $(1 / \mathrm{S})) \% * \%$ dV_dmu

\# sensitivity of e-dagger with respect to mortality change deta_dagger_dmu <- (kronecker $(\mathrm{t}(\mathrm{B}[, 1]) \% * \%$ t $(\mathrm{N}), \mathrm{t}($ mean_eta $))+$ kronecker $(t(N[, 1]),(t($ mean_eta) $\% * \% B))) \% * \%$ dvecU_dmu + $\operatorname{kronecker}(\mathrm{t}(\mathrm{N}[, 1]), \mathrm{t}($ mean_eta $)) \% * \%$ dvecM_dmu

\# sensitivity of Theil's with respect to mortality change $\mathrm{dT}_{-} \mathrm{dmu}<-\left(\mathrm{t}(\mathrm{x}) * \mathrm{eta}^{\wedge}(-1) * \log \left(\mathrm{t}(\mathrm{x}) * \mathrm{eta}^{\wedge}(-1)\right)\right) \% * \mathrm{df}_{-} \mathrm{dmu}-$ $\left(\mathrm{T} * \mathrm{eta}^{\wedge}(-1)+\mathrm{t}(\mathrm{f}) \% * \% \mathrm{x} * \mathrm{eta}^{\wedge}(-2)\right) \% * \%$ deta_dmu

\# sensitivity of MLD with respect to mortality change dMLD_dmu <- (t(e) * $\log ($ eta $)-\log (t(x))) \% * \%$ df_dmu + eta^ $(-1) \% * \%$ deta_dmu

\# sensitivity of Gini with respect to mortality change 


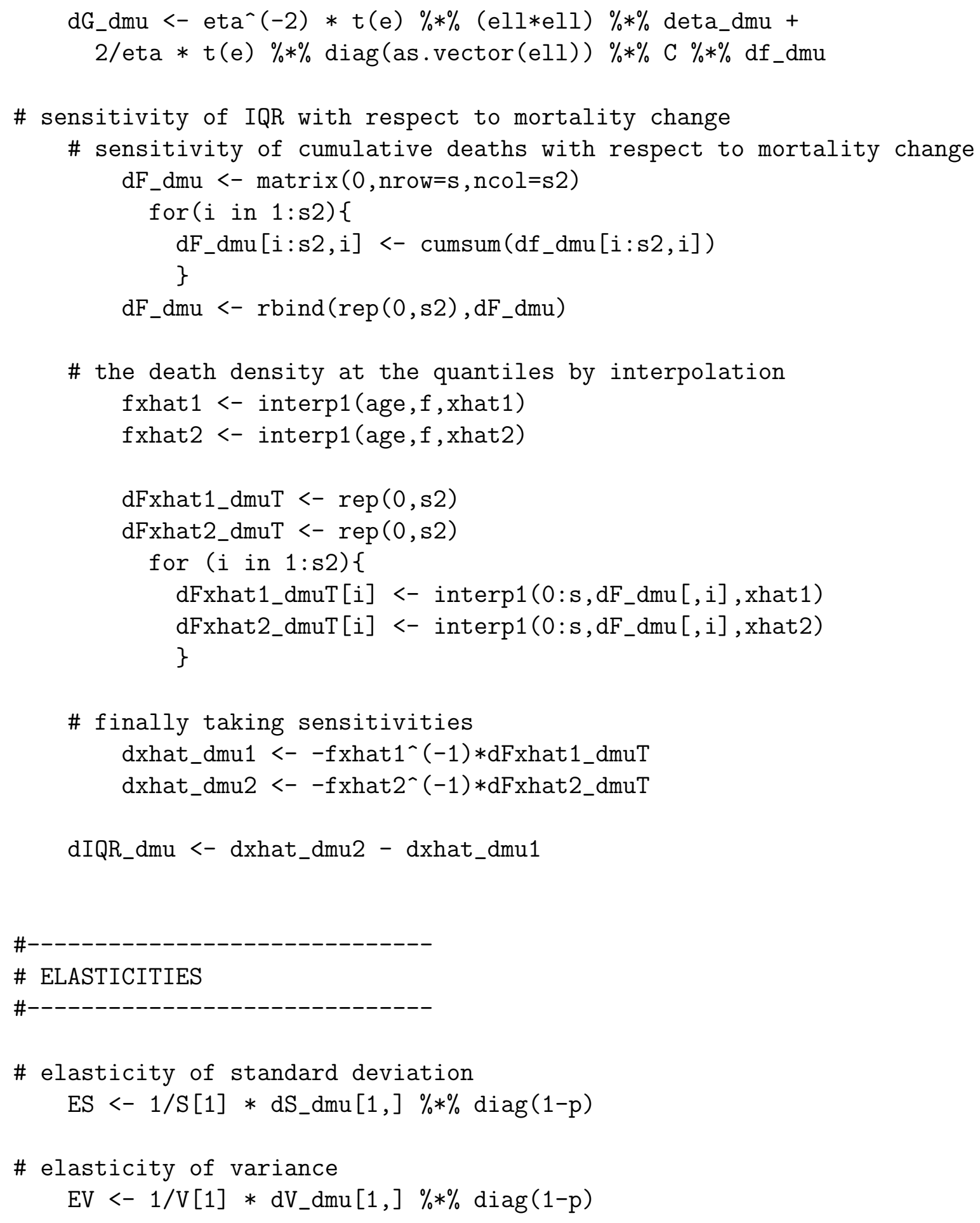




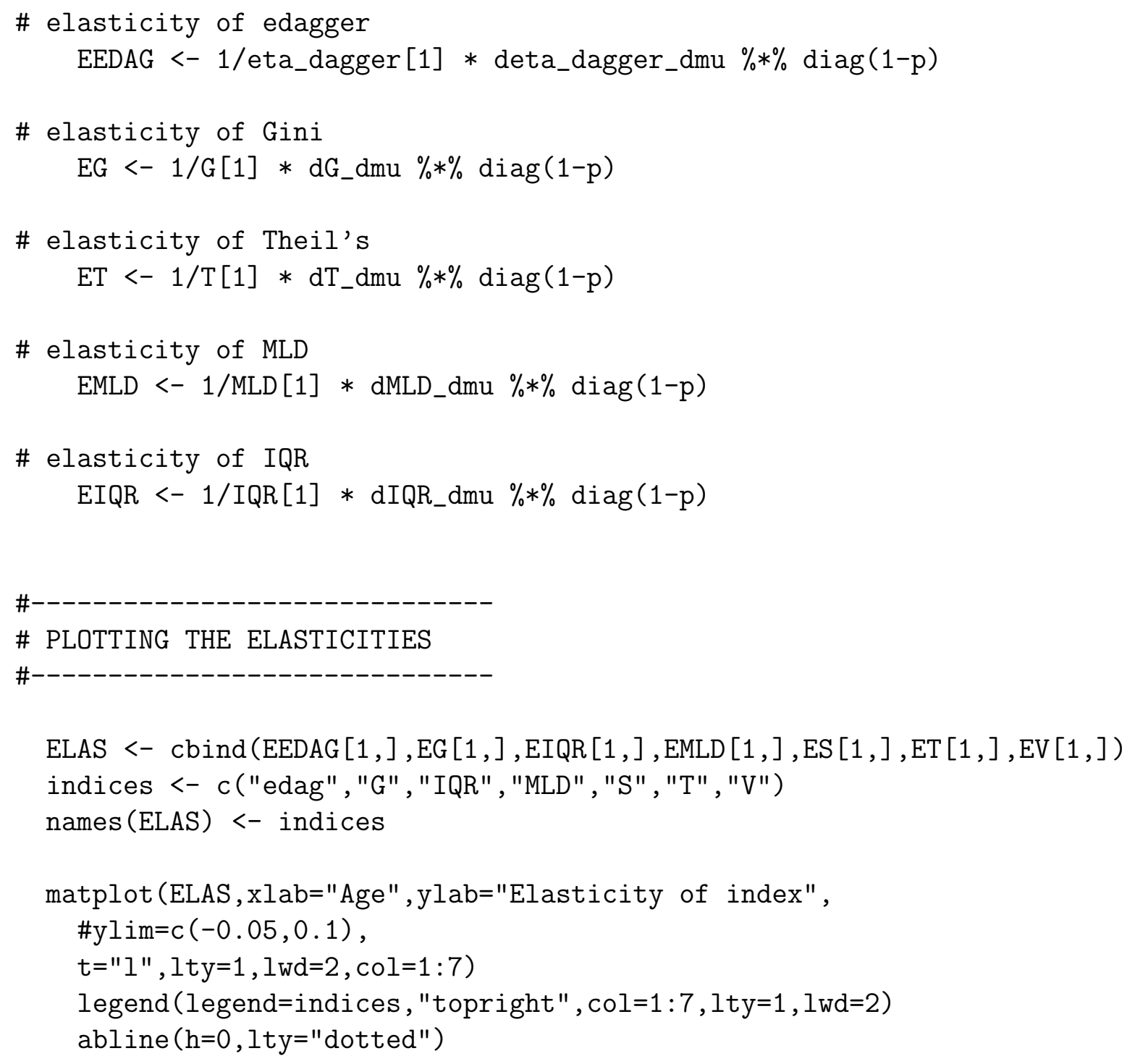




\section{References}

Abadir, K. M. and J. R. Magnus (2005). Matrix Algebra: Econometric Exercises. Cambridge University Press.

Allison, P. D. (1978). "Measures of Inequality." American Sociological Review 43:865-880.

Anand, S., F. Diderichsen, T. Evans, V. Shkolnikov and M. Wirth (2001). "Measuring disparities in health: Methods and indicators." In Challenging inequities in health: from ethics to action, pp. 49-67, New York: Oxford University Press.

Asada, Y. (2007). Health inequality: Morality and measurement. Toronto: University of Toronto Press.

Brown, D. C., M. Hayward, J. K. Montez, R. A. Hummer, C.-T. Chiu and M. M. Hidajat (2012). "The significance of education for mortality compression in the United States." Demography 49:819-840.

Caswell, H. (1978). "A general formula for the sensitivity of population growth rate to changes in life history parameters." Theoretical Population Biology 14:215 - 230.

(1989). "Analysis of life table response experiments I. Decomposition of effects on population growth rate." Ecological Modelling 46:221 - 237.

(2001). Matrix population models: Construction, analysis, and interpretation. 2nd ed., Sunderland, Massachusetts, USA.: Sinauer.

(2006). MAM2006: Markov Anniversary Meeting, chap. Applications of Markov chains in demography, pp. 319-334. Raleigh, North Carolina, USA,: Boson Books.

(2007). "Sensitivity analysis of transient population dynamics." Ecology Letters 10:1-15.

- (2008). "Perturbation analysis of nonlinear matrix population models." Demographic Research 18:59-116.

(2009). "Stage, age and individual stochasticity in demography." Oikos 118:1763-1782. 
(2010). "Perturbation analysis of longevity using matrix calculus." Paper presented at the 75th Annual Population Association of America meeting, in session 141: "Mathematical Aspects of Mortality and Longevity". Dallas, Texas .

(2011). "Sensitivity analysis of discrete Markov chains via matrix calculus." Linear Algebra and its Applications doi: 10.1016/j.laa.2011.07.046.

Cheung, S. L. and J. M. Robine (2007). "Increase in common longevity and the compression of mortality: the case of Japan." Population Studies 61:85-97.

Cheung, S. L., J. M. Robine, E. J. Tu and G. Caselli (2005). "Three dimensions of the survival curve: horizontalization, verticalization, and longevity extension." Demography 42:243-58.

Coale, A. J. (1972). The growth and structure of human populations: A mathematical investigation. Princeton University Press (Princeton, N.J).

Demetrius, L. (1969). "The sensitivity of population growth rate to pertubations in the life cycle components." Mathematical Biosciences 4:129 136.

Eakin, T. and M. Witten (1995). "How square is the survival curve of a given species?" Experimental Gerontology 30:33-64.

Edwards, R. D. and S. Tuljapurkar (2005). "Inequality in life spans and a new perspective on mortality convergence across industrialized countries." Population and Development Review 31:645-674.

Feichtinger, G. (1973). "Markovian models for some demographic processes." Statistical Papers 14:310-334.

Gakidou, E. and G. King (2002). "Measuring total health inequality: Adding individual variation to group-level differences." International Journal for Equity in Health 1:1-12.

Gakidou, E. E., C. J. L. Murray and J. Frenk (2000). "Defining and measuring health inequality: An approach based on the distribution of health expectancy." Bulletin of the World Health Organization 78:42-54. 
Goldman, N. and G. Lord (1986). "A new look at entropy and the life table." Demography 23:275-282.

Hamilton, W. (1966). "The moulding of senescence by natural selection." Journal of Theoretical Biology 12:12 - 45.

Herskind, A., M. McGue, N. Holm, T. Srensen, B. Harvald and J. Vaupel (1996). "The heritability of human longevity: A population-based study of 2872 Danish twin pairs born 18701900." Human Genetics 97(3):319-323.

HMD (2012). "Human Mortality Database. University of California, Berkeley (USA), and Max Planck Institute for Demographic Research (Germany). Available at www.mortality.org or www.humanmortality.de (data downloaded on $27 / 08 / 2012) . "$

Horiuchi, S., J. R. Wilmoth and S. D. Pletcher (2008). "A decomposition method based on a model of continuous change." Demography 45(4):785801.

Kannisto, V. (2000). "Measuring the compression of mortality." Demographic Research 3:1-24.

(2001). "Mode and dispersion of the length of life." Population: An English Selection 13:159-171.

Keyfitz, N. (1971). "Linkages of intrinsic to age-specific rates." Journal of the American Statistical Association 66:275-281. ley.

Keyfitz, N. and H. Caswell (2005). Applied Mathematical Demography. 3rd ed., New York, NY: Springer-Verlag.

Lambert, P. J. and J. R. Aronson (1993). "Inequality decomposition analysis and the Gini coefficient revisited." The Economic Journal 103:1221-1227.

Leon, D. A., L. Chenet, V. M. Shkolnikov, S. Zakharov, J. Shapiro, G. Rakhmanova, S. Vassin and M. McKee (1997). "Huge variation in Russian mortality rates 1984-94: Artefact, alcohol, or what?" The Lancet 350:383-388. 
Magnus, J. and H. Neudecker (1988). Matrix differential calculus with applications in statistics and econometrics. John Wiley \& Sons.

Magnus, J. R. and H. Neudecker (1985). "Matrix differential calculus with applications to simple, Hadamard, and Kronecker products." J. Math. Psychol. 29:474-492.

Marchand, S., D. Wikler and B. Landesman (1998). "Class, Health, and Justice." The Milbank Quarterly 76(3):449-467.

McGue, M., J. W. Vaupel, N. V. Holm and B. Harvald (1993). "Longevity is moderately heritable in a sample of Danish twins born 1870-1880." The Journal of Gerontology 48(6):237-244-.

Mookherjee, D. and A. Shorrocks (1982). "A decomposition analysis of the trend in UK income inequality." The Economic Journal 92:886-902.

Ouellette, N. and R. R. Bourbeau (2011). "Changes in the age-at-death distribution in four low mortality countries: A nonparametric approach." Demographic Research 25:595-628.

Pollard, J. (1982). "The expectation of life and its relationship to mortality." Journal of the Institute of Actuaries 109:225-240.

(1988). "On the decomposition of changes in expectation of life and differentials in life expectancy." Demography 25:265-276.

Roemer, J. E. (1996). Theories of distributive justice. Harvard University Press.

Roth, W. (1934). "On direct product matrices." Bulletin of the American Mathematical Society 40:461-468.

Sen, A. (1992). Inequality Reexamined. New York : Russell Sage Foundation ; Cambridge, Mass. : Harvard University Press, 1997.

Shkolnikov, V., E. Andreev and A. Z. Begun (2003). "Gini coefficient as a life table function. Computation from discrete data, decomposition of differences and empirical examples." Demographic Research 8:305-358.

Shorrocks, A. F. (1980). "The class of additively decomposable inequality measures." Econometrica 48:613-625. 
Smits, J. and C. Monden (2009). "Length of life inequality around the globe." Social Science \& Medicine 68:1114-1123.

Thatcher, A. R., S. L. K. Cheung, S. Horiuchi and J.-M. Robine (2010). "The compression of deaths above the mode." Demographic Research 22:505538 .

Theil, H. (1967). Amsterdam: North-Holland Publishing Co.

van Raalte, A. A. (2011). Lifespan variation: Methods, trends and the role of socioeconomic inequality. Ph.D. thesis, Erasmus University.

van Raalte, A. A., A. E. Kunst, P. Deboosere, M. Leinsalu, O. Lundberg, P. Martikainen, B. H. Strand, B. Artnik, B. Wojtyniak and J. P. Mackenbach (2011). "More variation in lifespan in lower educated groups: evidence from 10 European countries." International Journal of Epidemiology 40:1703-1714.

Vaupel, J. W. (1986). "How change in age-specific mortality affects life expectancy." Population Studies 40:147-157.

Vaupel, J. W. and V. Canudas Romo (2003). "Decomposing change in life expectancy: A bouquet of formulas in honor of Nathan Keyfitz's 90th birthday." Demography 40:201-216.

Vaupel, J. W., Z. Zhang and A. A. van Raalte (2011). "Life expectancy and disparity." BMJ Open 1:e000128. doi:10.1136/bmjopen-2011-000128.

Wagner, P. (2010). "Sensitivity of life disparity with respect to changes in mortality rates." Demographic Research 23(3):63-72.

WHO (2000). "The World Health Report 2000."

Willekens, F. J. (1977). "Sensitivity analysis in multiregional demographic models." Environment and Planning A 9:653-674.

Wilmoth, J. R. and S. Horiuchi (1999). "Rectangularization revisited: Variability of age at death within human populations." Demography 36:475495.

Wrycza, T. and A. Baudisch (2012). "How life expectancy varies with perturbations in age-specific mortality." Demographic Research 27:365-375. 
Zhang, Z. and J. W. Vaupel (2009). "The age separating early deaths from late deaths." Demographic Research 20:721-730. 


\section{Appendix figures depicting the sensitivity and elasticity of indices calculated from age 10}
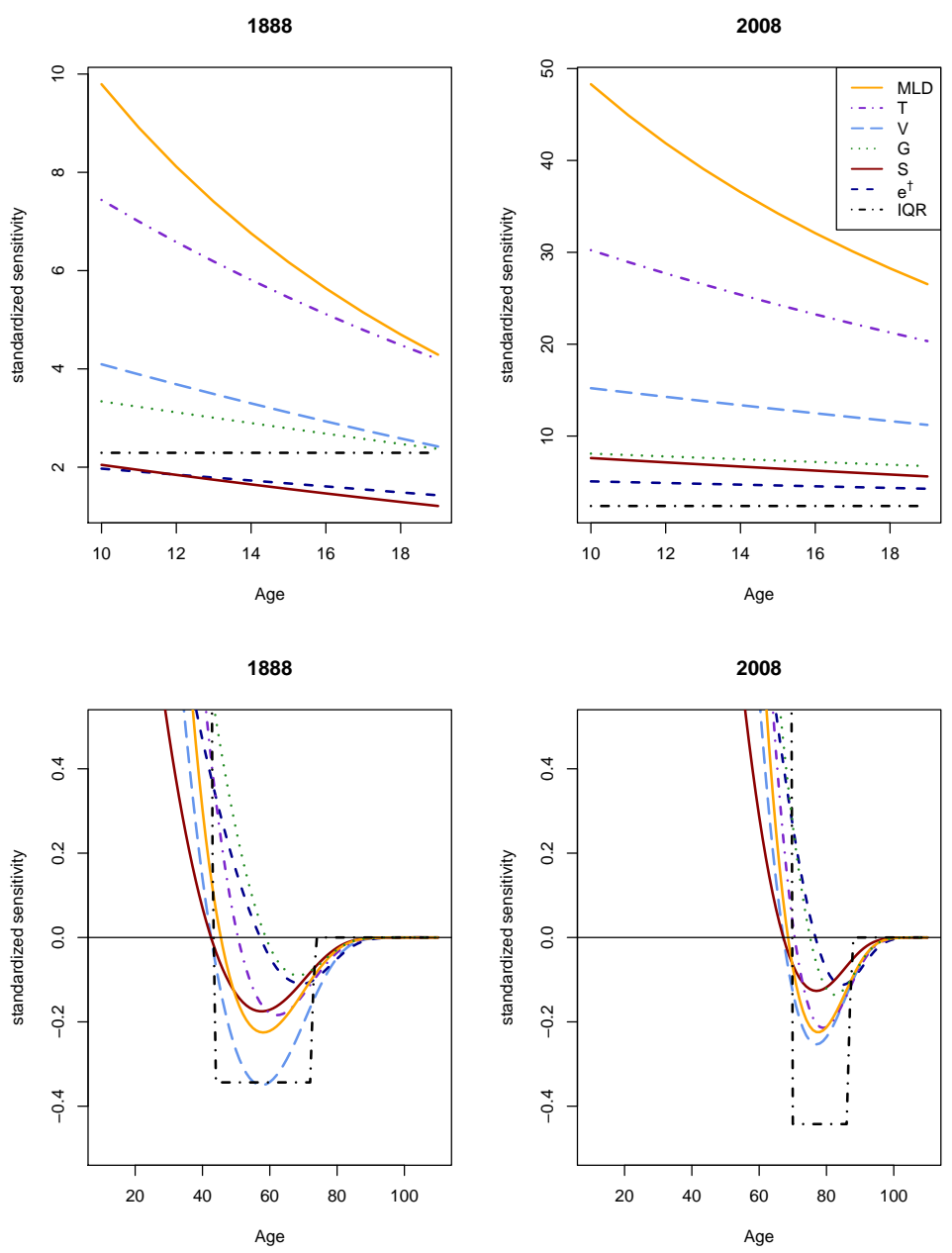

Figure 6: The sensitivity of each index conditional upon survival to age 10 with respect to mortality change at different ages. The sensitivities were standardized to the value of each index, i.e. $y_{10} \frac{d y}{d \theta}$, to make them comparable. Note the difference in scale between the top and bottom panels, plotted separately to more clearly delineate behaviour of the indices at early and later ages. French males, period lifetable data from the HMD. 
1888

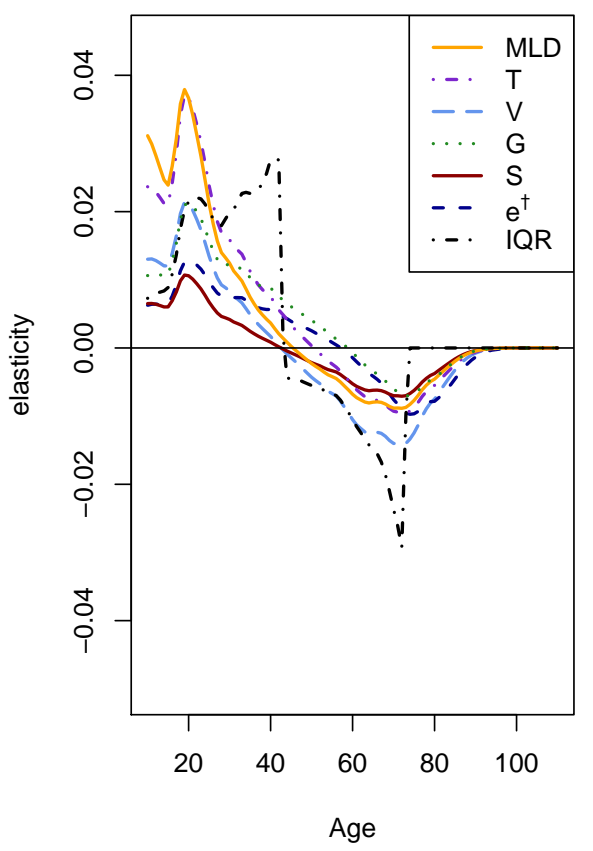

2008

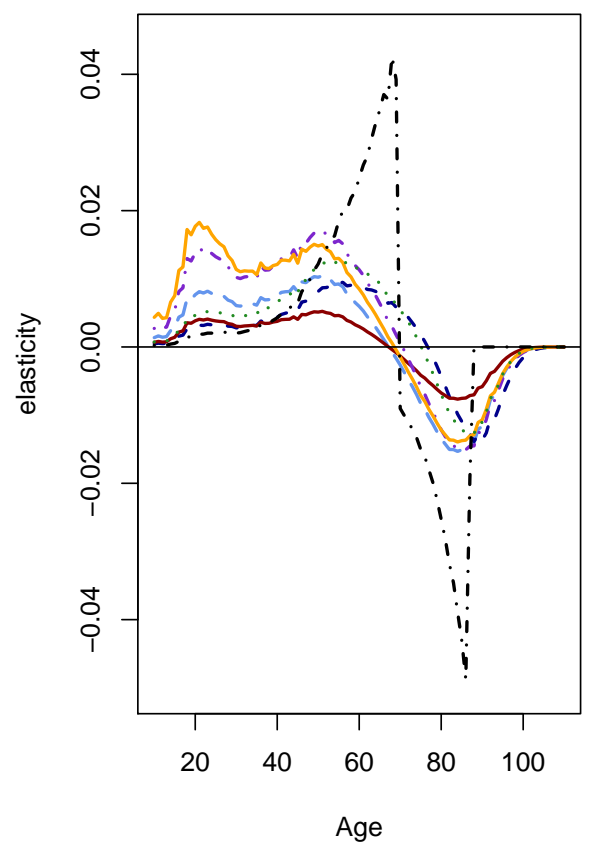

Figure 7: The proportional change in the index calculated conditional upon survival to age 10 from a one percent change in mortality at each age on the $\mathrm{x}$ axis. French males, period lifetable data from the HMD. 